\title{
Gleichheit durch Gleichstellungsmaßnahmen?
}

\section{Betriebliche Mentoringprogramme, Frauenquoten und geschlechtsspezifische Einkommensungleichheiten in Großbetrieben}

\author{
Eileen Peters $\cdot$ Jil Drobe $\cdot$ Anja Abendroth
}

Online publiziert: 24. Juli 2020

(C) Der/die Autor(en) 2020

Zusammenfassung Dieser Beitrag untersucht, inwiefern Mentoringprogramme und betriebliche Frauenquoten als zwei zentrale Gleichstellungsmaßnahmen mit geringeren geschlechtsspezifischen Einkommensungleichheiten in deutschen Großbetrieben einhergehen. Zusätzlich wird der Frage nachgegangen, inwiefern die Bedeutung dieser Gleichstellungsmaßnahmen zwischen Beschäftigtengruppen mit unterschiedlichem Qualifikationsniveau variiert und ob traditionelle Geschlechterideologien innerhalb der Belegschaft die Effektivität von Gleichstellungsmaßnahmen konterkarieren. Auf Grundlage von Linked-Employer-Employee-Daten, die sowohl administrative als auch Befragungsdaten für 5072 Beschäftigte in 93 Betrieben beinhalten, wurden Regressionsanalysen mit betriebsbezogenen Fixed Effects berechnet. Die Ergebnisse zeigen, dass Einkommensbenachteiligungen von Frauen innerhalb von Betrieben nicht mit dem Angebot von Mentoringprogrammen variieren. Demgegenüber zeigen sich geringere Einkommensungleichheiten zwischen den Geschlechtern in Betrieben mit Frauenquote. Dies gilt allerdings nur für Beschäftigte mit hohem Qualifikationsniveau und in Betrieben, in denen gleichzeitig eher egalitäre Geschlechterideologien in der Belegschaft vorherrschen. In traditionellen Betriebskontexten bestehen trotz Frauenquote geschlechtsspezifische Einkommensungleichheiten zwischen Beschäftigten mit höherem Qualifikationsniveau.

E. Peters $(\bowtie) \cdot$ A. Abendroth

Universität Bielefeld

Universitätsstraße 25, 33615 Bielefeld, Deutschland

E-Mail: eileen.peters@uni-bielefeld.de

A. Abendroth

E-Mail: anja.abendroth@uni-bielefeld.de

J. Drobe

Gütersloh, Deutschland 
Schlüsselwörter Einkommen · Arbeitsmarkt · Geschlechterideologie ·

Organisationale Ungleichheit · Geschlechterungleichheiten

\title{
Equality Through Equal Opportunity Policies?
}

Workplace Mentoring Programs, Female Quotas, and the Gender Pay Gap Within German Workplaces

\begin{abstract}
This article examines the extent to which mentoring programs and female quotas, as two central diversity policies, are associated with smaller gender wage gaps within large German workplaces. Furthermore, we investigated whether the implications of these diversity policies vary among workers with different levels of qualification and whether they are counteracted by traditional gender ideologies within the workforce. Using linked employer-employee data consisting of administrative and survey data for 5072 employees and 93 workplaces, we estimated fixed effect regression models. The results suggest that within workplaces, gender wage gaps are not associated with the presence of mentoring programs. In contrast, there is a substantial gender wage gap between women and men with higher qualification levels in workplaces without female quotas, whereas women earn similar wages as their male counterparts in workplaces with female quotas. However, this is only true if egalitarian gender ideologies are dominant within the workforce as we detect significant income disparities in traditional workplace contexts despite a female quota.
\end{abstract}

Keywords Income - Labour market · Gender ideologies · Organizational inequality $\cdot$ Gender inequalities

\section{Einleitung}

Frauen haben in den letzten Jahren sowohl hinsichtlich der Erwerbstätigenquote $(52,8 \%)$, die im Jahr 2016 annähernd so hoch war wie die der Männer (61,3\%), als auch bezüglich des Qualifikationsniveaus deutlich aufgeholt und Männer zum Teil sogar überholt (Helbig 2012; Statistisches Bundesamt 2018a, 2018b). So erlangen Frauen häufiger das Abitur als Männer (Helbig 2012) und in der Gruppe der 30bis 34-Jährigen häufiger einen Hochschulabschluss (27\%) (Statistisches Budensamt 2018c). Dennoch bestehen persistente geschlechtsspezifische Unterschiede in der Entlohnung sowie in der Repräsentation von Frauen in höheren Managementpositionen (Achatz et al. 2005; Abendroth et al. 2017; Steffes et al. 2017; Redmond und Mcguinness 2018; Holst und Wrohlich 2019). Generell gilt: je höher die betrachtete Hierarchieebene, desto geringer der Frauenanteil (Holst und Wiemer 2010; Holst und Wrohlich 2019).

Um die persistenten Ungleichheiten abzubauen, wurde im Jahr 2006 das Allgemeine Gleichbehandlungsgesetz (AGG) verabschiedet, das Benachteiligungen aufgrund des Geschlechts, ethnischer Herkunft, Rasse, Religion, Weltanschauung, Behinderung, Alter oder sexueller Identität entgegenwirken soll. Das AGG gibt Beschäftigten die rechtliche Grundlage, um Schadensersatz oder Entschädigungen von 
ihrem Arbeitgeber einzufordern, wenn eine Benachteiligung stattgefunden hat. Hierdurch ist der Druck auf deutsche Betriebe gestiegen, die Gleichberechtigung zwischen den Beschäftigten zu fördern (Aretz und Hansen 2003; Dreas 2019). Aufgrund der höheren Mitarbeiterheterogenität müssen sich Betriebe zudem vermehrt mit der externen und internen Vielfalt in Form von Diversitätsmanagement auseinandersetzen (Kirton und Greene 2005; Wrench 2007; Aretz und Hansen 2003). Der Ursprung des Diversitätsmanagements liegt im US-amerikanischen Kontext, wo Diversitätsmaßnahmen ${ }^{1}$ angewendet werden, um Diskriminierung entgegenzuwirken und somit die höchst mögliche Effektivität der Organisation zu erreichen (Dobbin et al. 1993; Naff und Kellough 2003; Kalev et al. 2006). Bisher wurden in Deutschland hingegen nur vereinzelt Maßnahmen zur Förderung von Vielfalt umgesetzt. Erst mit Inkrafttreten des AGG hat das Diversitätsmanagement in Deutschland an Bedeutung gewonnen (Merklein 2017). Neben Vereinbarkeits- oder Integrationsmaßnahmen sind auch Gleichstellungsmaßnahmen, die speziell für die Gleichstellung von Frauen und Männern am Arbeitsplatz entwickelt wurden, Teil des Diversitätsmanagements. Bisherige Forschung konnte bereits belegen, dass bestimmte Gleichstellungsmaßnahmen, die darauf abzielen, die Karrierenachteile von Frauen auszugleichen, erfolgreich die Diversität in Managementpositionen steigern (Kalev et al. 2006). Ein höherer Frauenanteil in Managementpositionen und in Betriebsvorständen begünstigt wiederum das berufliche Fortkommen aller weiblichen Beschäftigten (Cohen et al. 1998; Kurtulus und Tomaskovic-Devey 2012; Stainback et al. 2016) und verringert geschlechtsspezifische Einkommensunterschiede (Hultin und Szulkin 1999; Cohen und Huffman 2007; Cardoso und Winter-Ebmer 2010; Abendroth et al. 2017). Andererseits zeigt die Forschung, dass nicht alle Gleichstellungsmaßnahmen ihr Ziel erreichen (Kalev et al. 2006), wodurch deutlich wird, dass eine differenzierte Betrachtung der einzelnen Maßnahmen notwendig ist, um Aussagen über ihre Effektivität treffen zu können. Trotz der hohen Relevanz und Kosten für die Umsetzung von betrieblichen Gleichstellungsmaßnahmen wurde ihr Einfluss im wissenschaftlichen Diskurs für den deutschen Kontext selten empirisch erforscht (Ausnahme: Huffman et al. 2017). Dabei besteht gerade in Deutschland Handlungsbedarf, Wege zu finden, um die Chancengleichheit zwischen Frauen und Männern zu fördern. Denn auch im europäischen Vergleich fallen die Einkommensbenachteiligungen in Deutschland besonders hoch aus (Eurostat 2019). ${ }^{2}$ Erste Ergebnisse zeigen, dass das generelle Angebot von Gleichstellungsmaßnahmen mit geringeren geschlechtsspezifischen Einkommensungleichheiten in den unteren zwei Dritteln der Einkommensverteilung einhergeht (Huffman et al. 2017). Allerdings bleibt unklar, auf welche der implementierten Gleichstellungsmaßnahmen die geringeren Einkommensunterschiede zurückzuführen sind und inwiefern die vorherrschende Kultur im Betrieb eine Rolle für die Wirksamkeit der Gleichstellungsmaßnahmen spielt.

Das Ziel des vorliegenden Beitrages besteht darin, eine differenziertere Analyse der Relevanz von Gleichstellungsmaßnahmen für geschlechtsspezifische Einkom-

\footnotetext{
${ }^{1}$ Im englischsprachigen Kontext und vor allem in den USA oftmals als Equal Opportunity Policies bezeichnet.

2 Im Jahr 2017 hat Deutschland mit einer unbereinigten Lohnlücke von $21 \%$ den drittletzten Platz im europäischen Vergleich besetzt (Eurostat 2019).
} 
mensungleichheiten innerhalb von Großbetrieben vorzunehmen. Hierfür unterscheiden wir zwischen zwei zentralen Gleichstellungsmaßnahmen, die auf eine bessere Positionierung von Frauen in Betriebshierarchien abzielen: Mentoringprogramme und betriebliche Frauenquoten. Durch den Fokus auf innerbetriebliche Einkommensungleichheiten folgen wir einer organisationalen Ungleichheitsperspektive, die betont, dass die Produktion und Verteilung von Betriebsressourcen, wie Einkommen und Positionen, in Betrieben verhandelt wird (Baron und Bielby 1980; Reskin 2000; Tomaskovic-Devey und Avent-Holt 2019; Castilla 2008; Kalev 2009). Diese zeichnen sich durch eigene Ungleichheitsregime aus (Acker 2006; TomaskovicDevey und Avent-Holt 2019). Im Rahmen dieser Perspektive thematisieren wir verschiedene Mechanismen der Ungleichheitsgenese. Wir differenzieren zum einen, inwiefern diese beiden Gleichstellungsmaßnahmen mit einer besseren Positionierung von Frauen in der Betriebshierarchie einhergehen und so zur Verringerung der geschlechtsspezifischen Einkommensungleichheiten beitragen. Zum anderen analysieren wir, inwieweit die beiden Gleichstellungsmaßnahmen mit geringeren bereinigten Einkommensungleichheiten, die häufig durch Stereotypisierungsprozesse von Frauen als weniger produktiv begründet werden (Acker 1990; Ridgeway und Correll 2004; Risman und Davis 2013), assoziiert sind. Somit lassen sich zum einen Schlussfolgerungen darüber treffen, ob Gleichstellungsmaßnahmen zur Verbesserung der beruflichen Stellung von weiblichen Beschäftigten im Betrieb beitragen. Zum anderen können Rückschlüsse über den Abbau von Stereotypisierungsprozessen geschlossen werden, wenn der positive Zusammenhang auch für bereinigte Einkommensungleichheiten besteht. Zudem analysieren wir, ob die Wirksamkeit der beiden Maßnahmen zwischen Beschäftigtengruppen mit unterschiedlichem Qualifikationsniveau variiert. Gleichstellungsmaßnahmen sind häufig auf die Förderung von hochqualifizierten Frauen ausgerichtet (Berrey 2014; Mun und Jung 2018). Allerdings kann eine Veränderung der geschlechtsspezifischen Ungleichheiten in den oberen Rängen der Betriebshierarchie positive Auswirkungen auf weibliche Beschäftigte in unteren Statusgruppen haben (Cohen und Huffman 2007; Abendroth et al. 2017; Stojmenovska 2019).

In der Literatur gibt es weiterhin Befunde dafür, dass die Effektivität von Gleichstellungsmaßnahmen von der Akzeptanz und Unterstützung in der Belegschaft abhängig ist (Kidder et al. 2004; Kaplan 2006; Kalev et al. 2006; Dobbin und Kalev 2016; Scarborough et al. 2019). Hierauf aufbauend betrachten wir die Verbreitung von Geschlechterideologien, Einstellungen zur geschlechtsspezifischen Arbeitsteilung in der Belegschaft und untersuchen, inwiefern diese den Zusammenhang von Mentoringprogrammen sowie Frauenquoten mit geschlechtsspezifischen Einkommensungleichheiten mitbestimmen. Bisherige Forschung konnte bereits nachweisen, dass familienpolitische Maßnahmen, wie Elternzeit oder öffentliche Kinderbetreuung, je nach kultureller Unterstützung für die Berufstätigkeit von Müttern positive oder negative Zusammenhänge mit dem Einkommen von Müttern aufweisen (Budig et al. 2012). Daher entwickeln wir die These, dass die Effektivität von Gleichstellungsmaßnahmen durch die vorherrschenden Geschlechterideologien im Betrieb moderiert wird. Folglich lautet unsere Forschungsfrage: Inwiefern fallen geschlechtsspezifische Einkommensunterschiede in Großbetrieben mit Frauenquoten oder Mentoringprogrammen geringer aus und inwieweit variiert dieser Zusammen- 
hang in Abhängigkeit des Qualifikationsniveaus von Beschäftigten und den vorherrschenden Geschlechterideologien in der Belegschaft? Diese Forschungsfrage wird auf Grundlage eines Linked-Employer-Employee-Datensatzes (LEEP-B3), der sowohl administrative als auch Beschäftigten- und Betriebsinformationen verbindet, untersucht. Die hierarchische Datenstruktur ermöglicht die Zuordnung der Beschäftigten zu den Betrieben, wodurch innerbetriebliche Einkommensunterschiede analysiert und unter anderem geschlechtsspezifische Selektionsprozesse in unterschiedliche Betriebe ausgeschlossen werden können (Pausch et al. 2014; Abendroth et al. 2014; Diewald et al. 2014).

\section{Theoretischer Rahmen: Einkommensungleichheiten in Betrieben und Gleichstellungsmaßnahmen}

Geschlechtsspezifische Einkommensungleichheiten werden in der Forschung häufig über die berufliche Geschlechtersegregation am Arbeitsmarkt erklärt (Trappe und Rosenfeld 2004; Fitzenberger und Kunze 2005; Leuze und Strauß 2009; Kunze 2005; Boll et al. 2015). Diese lassen sich neben der geschlechtsspezifischen Bildungs- und Berufswahl auch durch einen erschwerten Zugang zu Berufen mit hohen Verdienstchancen für Frauen (gläserne Decke) sowie eingeschränkten Möglichkeiten des beruflichen Aufstiegs für frauendominierte Berufe mit relativ geringem Einkommen (klebriger Boden) begründen (Gallego und Wrohlich 2018). Die geschlechtsspezifische Arbeitsteilung, insbesondere nach Familiengründung, trägt dazu bei, dass die Erwerbsverläufe von Frauen eher Erwerbsunterbrechungen sowie Teilzeitbeschäftigung aufweisen. Hierdurch werden die Chancen auf den Zugang zu gut bezahlten Positionen eingeschränkt und es entstehen Stereotypisierungen gegenüber Frauen als weniger produktiv (Gangl und Ziefle 2009; Boll 2010; Ehrlich et al. 2019; Ejrnœs und Kunze 2013; Dechant und Blossfeld 2015). Personen neigen dazu, sich selbst und andere automatisch und unbewusst entlang sichtbarer und markanter Merkmale in Kategorien (Mann/Frau) einzuordnen (Tilly 1998; Taylor et al. 1978; Ridgeway 2011). Diesen Kategorien werden bestimmte Erwartungen hinsichtlich Fähigkeiten oder Produktivität zugeschrieben (Allport 1954; Devine 1989; Ridgeway et al. 1998; Ridgeway 2011), wodurch Personen und Betriebe den Wert einer Kategorie im Vergleich zu einer anderen Kategorie bewerten (Tilly 1998). Personen mit höherem Status verwenden diese kategorialen Unterschiede, um wichtige Betriebsressourcen, wie Einkommen und Positionen, für sich selbst oder Mitglieder ihrer Gruppe zu sichern, wodurch spezifische Möglichkeiten und Grenzen, je nach Gruppenzugehörigkeit, entstehen (DiTomaso et al. 2007; Stainback et al. 2016; Rivera 2012; DiTomaso 2013). Außerdem wird in der Literatur auf die Möglichkeit der Diskriminierung hingewiesen, wobei zwischen zwei Formen der Diskriminierung unterschieden wird. Die sogenannte „Taste-based“-Diskriminierung beschreibt, dass Individuen eine Gruppe gegenüber der anderen präferieren (Becker 1957). Die statistische Diskriminierung wird hingegen auf mangelnde Informationen über die Produktivität des Arbeitnehmers oder der Arbeitnehmerin zurückgeführt (Phelps 1972; Arrow 1973; Aigner und Cain 1977). Basierend auf Erfahrungswerten und gesellschaftlichen Rollenzuschreibungen wird jeder Gruppe eine durchschnittliche 
Arbeitsproduktivität zugewiesen. Da Frauen eine geringere Produktivität zugeschrieben wird, kann es zu Benachteiligungen hinsichtlich der Einstellungswahrscheinlichkeit, Beförderungsmöglichkeiten und Entlohnung kommen (Aigner und Cain 1977; Arrow 1973; Ridgeway und Correll 2004). Weiterhin bevorzugen Individuen, mit Mitgliedern aus derselben sozialen Gruppe zu interagieren (Ohlendieck 2003), was zu homogenen sozialen Netzwerken führt (Ibarra 1992; McPherson et al. 2001). Hieraus resultiert, dass Frauen zum einen durch soziale Schließungsprozesse (gläserne Decke) von bestimmten Positionen im Betrieb ausgeschlossen werden und ihnen zum anderen der Zugang zu höheren Löhnen vorenthalten wird (Acker 1990; Tomaskovic-Devey und Avent-Holt 2019). Wenn die Zuordnung zwischen Kategorien, wie Geschlecht und Status, mit bestehenden Ansichten und Einstellungen übereinstimmen, beispielsweise, wenn alle Führungspositionen mit Männern besetzt sind und alle anderen Tätigkeiten von Frauen ausgeübt werden, werden Ungleichheiten intensiviert (Tilly 1998; Ridgeway et al. 1998; Tomaskovic-Devey und AventHolt 2019). Demgegenüber fallen geschlechtsspezifische Ungleichheiten geringer aus, wenn die Zuordnung den Ansichten widerspricht (Statusinkonsistenz) und beispielsweise Frauen Führungspositionen innehaben (Abendroth et al. 2017). Neben nationalen und regionalen Unterschieden in der Lohnlücke zwischen Männern und Frauen (Mandel und Semyonov 2006; Arulampalam et al. 2007; Nisic 2017; Fuchs et al. 2019) weisen aktuelle Forschungsergebnisse in der Tat darauf hin, dass die unterschiedliche Selektion von Männern und Frauen in Betrieben zu geschlechtsspezifischen Einkommensungleichheiten beitragen (Ludsteck 2014; Fuchs et al. 2019; Frodermann et al. 2018). Einkommensungleichheiten variieren jedoch auch zwischen Betrieben (Achatz et al. 2005; Wolf und Heinze 2010; Gartner und Hinz 2009; Abendroth et al. 2017).

Welche Rolle spielt dabei die Umsetzung von Gleichstellungsmaßnahmen in Betrieben? Gleichstellungsmaßnahmen, und Diversitätsmaßnahmen generell, gelten als Vehikel zur Erreichung der Chancengleichheit in Arbeitsorganisationen (Jüngling und Rastetter 2011; Kandola und Fullerton 1994; Wrench 2007; Kirton und Greene 2005). Die Einführung dieser Gleichstellungsmaßnahmen kann dabei für Betriebe mit einem ökonomischen Nutzen verbunden sein. Diversität kann dazu beitragen, das Kreativitäts- und Innovationspotenzial in Betrieben zu fördern (Cox und Blake 1991; Buche et al. 2013; Krell und Sieben 2011; Dreas 2019), was wiederum zu Umsatz- und Gewinnsteigerung führen kann (Herring 2009). Zudem kann das Angebot von Gleichstellungsmaßnahmen dazu führen, dass das Ansehen als attraktiver Arbeitgeber erhöht wird, was insbesondere im Hinblick auf eine verstärkte Konkurrenzsituation um qualifizierte Arbeitskräfte für das Fortbestehen des Betriebes von großer Bedeutung ist (Iseke und Pull 2017). Bisherige Forschung hat bereits gezeigt, dass durch eine Veränderung der Betriebspolitiken und Personalstrategien, wie formalisierte Personalsysteme, Maßnahmen zur Förderung der Chancengleichheit und Unterstützungsleistungen bei der Kinderbetreuung, geschlechtsspezifische Einkommensungleichheiten in Betrieben abgebaut werden können (Wolf und Heinze 2010; Abendroth et al. 2017; Huffman et al. 2017). Weiterhin können durch den Abbau der Geschlechtersegregation entlang der Betriebshierarchie soziale Beziehungen und Kooperationen zwischen Frauen und Männern gefördert werden, wodurch die Einstellungen in der Belegschaft bezüglich der Zuschreibungen von Fähigkeiten 
und Status verändert werden (Kanter 1977; Pettigrew und Tropp 2006; Kalev 2009). Im Folgenden wird genauer erläutert, wie Mentoringprogramme und Frauenquoten, die zur Erreichung von Chancengleichheit für Frauen und Männer implementiert werden, Einkommensungleichheiten entgegenwirken können.

\subsection{Mentoringprogramme}

Im beruflichen Kontext werden Mentoringbeziehungen als eine Förderbeziehung zwischen einer meist jüngeren Person am Anfang ihrer beruflichen Laufbahn (Mentee) und einer erfahreneren Person (Mentor) verstanden (Graf und Edelkraut 2017). Der Mentor soll hier beratend und unterstützend agieren sowie Fach- und Erfahrungswissen an den Mentee weitergeben. Mentoren dienen weiterhin als Berufsberater und als Orientierungshilfe im Betrieb (Brown 1986). Ziel des Mentoring ist es, die persönliche und berufliche Entwicklung des Mentees zu fördern. Es lässt sich zwischen dem formellen Mentoring, das vom Betrieb initiiert wird, und dem informellen Mentoring, das ohne die Unterstützung des Betriebs zustande kommt, unterscheiden (Graf und Edelkraut 2017). Frauen haben unter anderem aufgrund vorhandener Betriebsstrukturen geringere Chancen als Männer, eine informelle Mentoringbeziehung einzugehen (Cox 1994; Ragins und Cotton 1999). Diese informellen Netzwerke von Männern führen häufig bei Rekrutierungsprozessen für Führungspositionen dazu, dass Frauen als potenzielle Kandidatinnen für diese Positionen erst gar nicht wahrgenommen und dementsprechend auch nicht ausreichend ge- und befördert werden. Daher wurde die gezielte Förderung von weiblichen Nachwuchskräften in Form von formellen Mentoringprogrammen etabliert, um die gläserne Decke für Frauen beim Zugang zu gut bezahlten Positionen zu durchbrechen (Kurmeyer und Höppel 2017). Mentoringprogramme ermöglichen Frauen den Zugang zu wichtigen Betriebsinformationen, beispielsweise über vakante Stellen, die ihre männlichen Kollegen durch die sogenannten „old-boy networks“ erhalten (Ragins 1989; Brown 1986; Kurmeyer und Höppel 2017). Die Beziehung zu einem statushöheren Mentor verbessert weiterhin die Position der weiblichen Beschäftigten sowie ihre Verhandlungsmacht im Betrieb, da mithilfe der Unterstützung des Mentors Forderungen erfolgreicher durchgesetzt werden können (Scandura 1992; Kurmeyer und Höppel 2017). Collins (1983) konnte feststellen, dass Frauen durch die Unterstützung und die Ermutigung ihrer Mentoren an Selbstbewusstsein gewinnen. Ferner zeigt Collins, dass die Sichtbarkeit der Mentees im Betrieb gesteigert wird, wodurch die Karrieremöglichkeiten von Frauen erhöht werden. Es besteht überdies ein positiver Zusammenhang zwischen Mentoringprogrammen und Einkommen, Jobzufriedenheit und beruflichem Fortkommen (Burke 1984; Fragenson 1988; Whitely et al. 1991). So können Mentoringprogramme einerseits die berufliche Geschlechtersegregation im Betrieb reduzieren und so zu einer Reduzierung von geschlechtsspezifischen Einkommensungleichheiten beitragen. Andererseits können, im Sinne sozialer Kontakttheorien, durch den geförderten Austausch zwischen Frauen und Männern auf verschiedenen Hierarchieebenen geschlechtsspezifische Stereotype zur Arbeitsproduktivität von Frauen abgebaut werden (Kurmeyer und Höppel 2017; Pettigrew 1998; Şherif 1966; Kalev 2009). Darauf aufbauend kann von zusätzlichen Einflüssen von Mentoringprogrammen auf geschlechtsspezifische Einkommensungleichheiten 
unabhängig von der Bedeutung für die berufliche Geschlechtersegregation ausgegangen werden. Wir nehmen an:

Hla In Betrieben, in denen Mentoringprogramme angeboten werden, fallen die geschlechtsspezifischen Einkommensunterschiede geringer aus.

\subsection{Freiwillige Frauenquote}

Als Erklärung für die persistente Unterrepräsentation von Frauen in Führungspositionen wird oftmals angeführt, dass Frauen in der „Pipeline“ stehen und in Zukunft Führungspositionen in Unternehmen einnehmen werden. Der rasante Anstieg von Hochschulabsolventinnen spiegelt sich allerdings nicht in dem nur marginalen Anstieg des Frauenanteils in Spitzenpositionen der Wirtschaft wider (Holst und Wiemer 2010). Unter anderem ist dies darauf zurückzuführen, dass Individuen es bevorzugen, mit Personen, die ihnen ähnlich und Mitglieder ihrer Gruppe sind (z. B. Geschlecht, Religion, Herkunft), zu interagieren und sie als vertrauenswürdiger, kompetenter und zuverlässiger einstufen (Tajfel und Turner 1979; Fiske 1998; Reskin 2000; Ibarra 1992; McPherson et al. 2001). Da Männer traditionell Macht- und Autoritätspositionen im Betrieb besetzten, werden somit ihre Dominanz und der Status quo aufrechterhalten (Kanter 1977; Elliott und Smith 2004; McPherson et al. 2001; Ibarra 1992). Diesen Prozessen kann eine bevorzugte Stellenbesetzung zugunsten von Frauen entgegenwirken. Frühere Studien haben bereits aufgezeigt, dass sich ein höherer Frauenanteil im mittleren und gehobenen Management positiv auf das Einkommen aller weiblichen Beschäftigten auswirkt sowie die Wahrscheinlichkeit einer Beförderung erhöht (Hultin und Szulkin 1999; Cohen und Huffman 2007; Krishnan und Parsons 2008; Cardoso und Winter-Ebmer 2010). Hirsch (2013) hat für Deutschland festgestellt, dass eine Erhöhung des Frauenanteils im gehobenen und mittleren Management die unerklärten geschlechtsspezifischen Einkommensungleichheiten innerhalb des Betriebes verringert. Des Weiteren wirkt sich die prozentuale Erhöhung des Frauenanteils im Managementbereich positiv auf die untergeordnete weibliche Statusgruppe aus. Dies führt zu einer Aufwertung der Kategorie „Frau“ und zu einem höheren Einkommen aller Frauen im Betrieb. Zudem zeichnet sich ab, dass ein erhöhter Frauenanteil im Management auch zu einer erhöhten Befürwortung der Chancengleichheit führt und andere Frauen bei dem Zugang zu guten Positionen unterstützt werden (Cohen et al. 1998). Die Beförderungswahrscheinlichkeit steigt bereits, sobald nur eine Frau eine Position in der nächsthöheren Hierarchieebene besetzt. Darüber hinaus können Frauen in Managementpositionen zeigen, dass Frauen ebenso kompetent und fähig in der Ausübung von Managementpositionen sind wie Männer und als positive Rollenvorbilder für andere Frauen fungieren (Ely 1995; Singh et al. 2006; Lockwood 2006). Darauf aufbauend kann davon ausgegangen werden, dass durch Gleichstellungsmaßnahmen zum einen die Geschlechtersegregation im Betrieb abgebaut wird, da Frauen einen besseren Zugang zu Positionen im Betrieb erhalten, wodurch die geschlechtsspezifischen Einkommensungleichheiten geringer ausfallen sollten. Zum anderen können über die bessere Positionierung und dem resultierenden Abbau der Geschlechtersegregation, der zu Statusinkonsistenzen führt, bestehende Stereotype und Ansichten gegenüber Frauen als weniger 
produktiv, hin zu engagiert oder kompetent geändert werden (Kanter 1977; Tomaskovic-Devey und Avent-Holt 2014). Folglich argumentieren wir, dass sowohl die unbereinigten als auch bereinigten Einkommensungleichheiten in Betrieben, in denen Frauenquoten angeboten werden, geringer ausfallen. Wir formulieren folgende Hypothese:

$H 1 b$ In Betrieben, in denen die Förderung von Frauen durch eine betriebliche Frauenquote gesichert ist, fallen die geschlechtsspezifischen Einkommensunterschiede geringer aus.

Jedoch ist davon auszugehen, dass die betriebliche Frauenquote einen stärkeren $\mathrm{Zu}$ sammenhang mit geschlechtsspezifischen Einkommensungleichheiten aufweist als Mentoringprogramme, da diese Maßnahme direkt in die Beförderungsstrukturen im Betrieb eingreift, sodass Frauen bei gleicher Qualifikation bevorzugt werden müssen. Hierdurch wird die Transparenz in Beförderungsprozessen erhöht, da Verantwortliche ihre Entscheidungen rechtfertigen müssen und die individuelle Handlungsfreiheit nach eigenem Ermessen von Entscheidungsträgern eingeschränkt wird. So wird direkt in Strukturen des Personalmanagements eingegriffen. Mentoringprogramme unterstützen Frauen zwar darin, ihre sozialen Netzwerke auszubauen und soziale Kontakte zu statushöheren Individuen zu knüpfen, aber dies muss nicht zwangsläufig dazu führen, dass sie tatsächlich im Beförderungsprozess bevorzugt werden.

H1c Betriebliche Frauenquoten sind stärker mit geringeren geschlechtsspezifischen Einkommensungleichheiten assoziiert als Mentoringprogramme.

\subsection{Die Bedeutung von Gleichstellungsmaßnahmen entlang des Qualifikationsniveaus}

Gleichstellungsmaßnahmen wie Mentoringprogramme und Frauenquoten sind größtenteils an Frauen mit hohem Qualifikationsniveau adressiert und verfolgen das Ziel, den Frauenanteil in Führungspositionen zu erhöhen (Kaiser-Belz 2008; Frodermann et al. 2018; Berrey 2014; Mun und Jung 2017). Insbesondere festgelegte Frauenquoten fokussieren meist die Erhöhung des Frauenanteils in Positionen am oberen Ende der Betriebshierarchie (Bertrand et al. 2018; Maume et al. 2019). Durch die Implementierung einer Frauenquote erhalten hochqualifizierte Frauen zum einen den Zugang zu hohen Positionen im Betrieb, wodurch die geschlechtsspezifische Lohnlücke verringert werden kann. Zum anderen können die Einkommensunterschiede innerhalb derselben Position durch Statusinkonsistenzen verringert werden. Die Erwartungen und Einschätzungen hinsichtlich der Arbeitsproduktivität und Kompetenz von Frauen werden angepasst, wodurch ebenfalls die bereinigte Lohnlücke geringer ausfallen sollte. Dies wird durch bisherige Forschung unterstützt, die nachweist, dass eine Frauenquote für Betriebsvorstände nicht nur den Frauenanteil in Vorständen erhöht, sondern darüber hinaus die geschlechtsspezifischen Einkommensunterschiede zwischen männlichen und weiblichen Vorstandsvorsitzenden verringert (Bertrand et al. 2018; Maume et al. 2019). Allerdings kann eine geringere Geschlechtersegregation in Führungspositionen einen sogenannten „Trickle-down“-Effekt nach sich 
ziehen, sodass auch Frauen, die nicht direkt durch diese Maßnahmen betroffen sind, eine bessere Positionierung sowie Entlohnung erhalten (Huffman et al. 2017). Der Abbau von Stereotypisierungen durch die bessere Positionierung von Frauen betrifft alle weiblichen Beschäftigten im Betrieb, und Frauen in höheren Positionen können sich für Frauen aus unteren Statusgruppen einsetzen und als ,,agents of change“ agieren (Cohen und Huffman 2007; Abendroth et al. 2017; Stojmenovska 2019). So mag ein positiver Zusammenhang für Frauen mit geringerem Qualifikationsniveau bestehen, allerdings sollte dieser schwächer ausfallen als für Frauen mit hohem Qualifikationsniveau, da sie die Hauptzielgruppe darstellen.

H1d Der positive Zusammenhang von Gleichstellungsmaßnahmen und geschlechtsspezifischen Einkommensungleichheiten fällt stärker für Beschäftigte mit hohem Qualifikationsniveau aus.

\subsection{Geschlechterideologien}

Neo-institutionalistische Perspektiven legen nahe, dass Betriebe nicht per se Gleichstellungsmaßnahmen anbieten, weil sie damit einen ökonomischen Nutzen verbinden, sondern weil sie durch einen gestiegenen Druck von außen durch die Implementierung von Gleichstellungsmaßnahmen Legitimität erhalten (Kelly und Dobbin 1999; Dobbin 2009). Betriebe passen ihre Praktiken entsprechend der institutionellen Umwelterwartungen an, was allerdings oftmals nur symbolisch geschieht (Meyer und Rowan 1977; DiMaggio und Powell 1983). Dies kann dazu führen, dass Gleichstellungsmaßnahmen nur leere Hüllen (,empty shells“) darstellen, sodass Gleichstellungsmaßnahmen nur der Außendarstellung gelten, die geschlechtsspezifischen Ungleichheiten innerhalb des Betriebs jedoch bestehen bleiben (Hoque und Noon 2004).

Die Einstellungen und Ansichten der Beschäftigten stellen einen wichtigen Einflussfaktor für die Effektivität von Gleichstellungsmaßnahmen dar. Studien konnten bereits nachweisen, dass Gleichstellungsmaßnahmen effektiver sind, wenn sie von Beschäftigten und Führungskräften unterstützt und gefördert werden (Kalev et al. 2006; Dobbin und Kalev 2016). Ob eine Gleichstellungsmaßnahme auf Unterstützung im Betrieb trifft oder nicht, ist weiterhin abhängig von der Vorgehensweise der jeweiligen Maßnahme sowie den Ansichten der Beschäftigten über Diskriminierung als Grund für die Entstehung von Ungleichheiten (Scarborough et al. 2019). Wir argumentieren, dass die Zustimmung der Beschäftigten zu traditionellen oder egalitären Geschlechterideologien die Intensität von Stereotypisierungsprozessen innerhalb der Betriebe widerspiegelt und somit die normativen Barrieren für Frauen innerhalb des Betriebs abbildet.

Als Geschlechterideologie wird allgemein der individuelle Unterstützungsgrad hinsichtlich einer geschlechtsspezifischen Aufgabenverteilung von bezahlter Arbeit und familiären Betreuungsaufgaben angesehen (Davis und Greenstein 2009). Neuere Studien zeigen, dass Geschlechterideologien verschiedene Dimensionen umfassen, die zwischen Länderkontexten sowie Frauen und Männern unterschiedlich ausgeprägt sein können und somit ein multidimensionales Konstrukt darstellen (Yu und 
Lee 2013; Knight und Brinton 2017; Grunow et al. 2018). ${ }^{3}$ Die Zustimmung zu traditionellen Geschlechterideologien kann den positiven Zusammenhang zwischen familienpolitischen Maßnahmen wie Elternzeit und Kinderbetreuungsmöglichkeiten und dem Einkommen von Müttern beeinflussen (Budig et al. 2012). Diese Beziehung zwischen Geschlechterideologien und staatlichen Politiken kann auch für die Betriebsebene und die hier implementierten Gleichstellungsmaßnahmen vermutet werden. In Betrieben, in denen traditionelle Geschlechterideologien in der Belegschaft dominieren, stoßen Gleichstellungsmaßnahmen auf mehr Resistenz, wodurch es unwahrscheinlicher wird, dass in diesen Betrieben Gleichstellungsmaßnahmen mit geringeren geschlechtsspezifischen Einkommensunterschieden einhergehen. Demgegenüber stoßen die Maßnahmen in Betrieben mit egalitären Geschlechterideologien auf weniger Barrieren und mehr Zustimmung. Dementsprechend formulieren wir folgende Hypothese:

H2 Der positive Zusammenhang von Gleichstellungsmaßnahmen und geschlechtsspezifischen Einkommensungleichheiten fällt geringer aus, wenn traditionelle Geschlechterideologien in der Belegschaft dominieren.

\section{Daten, Variablen und Methode}

\subsection{Daten}

Als Datengrundlage wird die erste Welle des Linked-Employer-Employee-Panels (LEEP-B3), die im Jahr 2012 erhoben wurde, verwendet (Abendroth et al. 2014; Diewald et al. 2014; Pausch et al. 2014). Dieser Datensatz wurde in dem Sonderforschungsbereich 882 ,Von Heterogenitäten zu Ungleichheiten“ im Projekt B3 „Wechselwirkungen zwischen Verwirklichungschancen im Berufs- und Privatleben: Eine Untersuchung von Beschäftigten in unterschiedlichen Arbeitsorganisationen“ an der Universität Bielefeld in Zusammenarbeit mit dem Institut für Arbeitsmarktund Berufsforschung (IAB) erhoben. Im Fokus der Untersuchung stehen Beschäftigte sowie ihre berufliche und private Situation (Abendroth et al. 2014; Diewald et al. 2014; Pausch et al. 2014). Hierbei soll untersucht werden, welche Rolle die Arbeitsumwelt in der Produktion von sozialer Ungleichheit spielt (Abendroth et al. 2014; Diewald et al. 2014; Pausch et al. 2014). Damit die gesammelten Informationen der Beschäftigten denen der Betriebe zugeordnet werden können, fand die Datenerhebung in einem zweistufigen Prozess statt.

Zuerst wurden, in Kooperation mit dem Forschungsdatenzentrum der Bundesagentur für Arbeit, die Betriebe auf Basis der administrativen Betriebsdaten des IAB gezogen (Pausch et al. 2014). Die Grundlage für die Stichprobenziehung stellte die Beschäftigtenhistorie des IAB dar. Hierbei wurden nur Betriebe, die mindestens 500 regulär beschäftigte Personen gemeldet hatten, in die Grundgesamtheit

\footnotetext{
3 Grunow et al. (2018) identifizieren mit einer latenten Klassenanalyse fünf vorherrschende Klassen von Geschlechterideologien, die von egalitär bis traditionell reichen.
} 
aufgenommen. ${ }^{4}$ Die Festlegung auf mindestens 500 Beschäftigte bietet den Vorteil, dass nur ein Teil der Belegschaft kontaktiert werden muss, um die antizipierte Anzahl von durchschnittlich 67 Beschäftigten pro Betrieb zu realisieren (Pausch et al. 2014). Somit können mögliche Befragungseffekte oder Reaktivität umgangen oder zumindest minimiert werden (Pausch et al. 2014). Um sicherzustellen, dass eine hohe Varianz der Betriebe in den verschiedenen Wirtschaftszweigen (WZ 2008) und eine ausreichend hohe Anzahl an ostdeutschen Betrieben im Sample enthalten sind, wurde eine disproportional geschichtete Zufallsstichprobe gezogen (Pausch et al. 2014; Diewald et al. 2014). Dies resultiert in einer Grundgesamtheit von 3934 Betrieben, die in 34 Schichten, nach Wirtschaftszweigen und Ost-/Westdeutschland, aufgeteilt wurden. ${ }^{6}$ Aus dieser Grundgesamtheit wurde eine Bruttostichprobe von 539 Betrieben gezogen, von denen in 115 Betrieben Interviews mit Personalverantwortlichen durchgeführt werden konnten (Pausch et al. 2014). Dies entspricht einer Rücklaufquote von 23,9\% (Pausch et al. 2014). Im Zuge der Betriebsbefragung wurden Informationen hinsichtlich der Formalstruktur, Personalpolitik sowie den implementierten Diversitätsmaßnahmen erhoben. Von den 115 Betrieben haben 15 nicht zugestimmt, dass ihre Beschäftigten für die Befragung kontaktiert werden dürfen (Pausch et al. 2014).

Anschließend wurden in diesen 100 Betrieben durchschnittlich 65 Beschäftigte interviewt, die zufällig aus allen sozialversicherungspflichtigen Beschäftigten gezogen wurden (Abendroth et al. 2014). Insgesamt konnten 6454 Hauptinterviews realisiert werden. Zusätzlich haben 2185 Partner und Partnerinnen der Hauptbefragten der Survey-Teilnahme zugestimmt. ${ }^{7}$ Bei der Befragung wurden Informationen über die Persönlichkeit der Beschäftigten, ihre Lebenseinstellungen, ihr Privatleben und ihre Arbeitsbedingungen erhoben. Befragte, die der Verknüpfung von administrativen Daten des IAB zugestimmt haben, wurden diese zugespielt. Diese Daten enthalten Informationen über die Beschäftigtenhistorie der Befragten, wie beispielsweise tagesgenaue Angaben darüber, seit wann die Beschäftigten in dem jetzigen Betrieb angestellt sind. Auf Grundlage von multivariaten Selektivitätsanalysen und dem Vergleich zur Grundgesamtheit kann sowohl für die Betriebs- als auch die Beschäftigtenbefragung festgehalten werden, dass sie nicht wesentlich von der Grundgesamtheit abweichen (Pausch et al. 2014; Abendroth et al. 2014). Damit ist das LEEP-B3 repräsentativ für deutsche Großbetriebe und Beschäftigte in Großbetrieben.

Unsere Analysen basieren auf Querschnittsdaten, wodurch keine kausalen Aussagen vorgenommen werden können. Vor diesem Hintergrund werden die Ergebnisse als Korrelationen und Zusammenhänge interpretiert, die nichtsdestotrotz auf-

\footnotetext{
${ }^{4}$ Diese Voraussetzung trifft auf 0,2\% aller Betriebe in Deutschland zu, in denen allerdings $19,1 \%$ aller Beschäftigten angestellt sind (Frodermann et al. 2018).

5 Die Einteilung der Wirtschaftszweige wurde anhand der Klassifikation der Wirtschaftszweige (WZ 2008) vorgenommen. Diese Klassifikation ist die gängigste Branchengliederung (Pausch et al. 2014). Ausgeschlossen wurden Wirtschaftszweige A, B, S, T und U, da diese Kontexte stark von Betriebskontexten der anderen Wirtschaftszweige abweichen (Pausch et al. 2014).

6 Unser Sample umfasst Betriebe aus dem privaten und öffentlichen Sektor.

7 Die Partnerinterviews bestanden aus einer verkürzten Version des Fragebogens für Hauptbefragte.
} 
schlussreiche Einblicke geben können, inwieweit geschlechtsspezifische Einkommensungleichheiten in Betrieben mit Gleichstellungsmaßnahmen assoziiert sind. Wir schließen alle Personen und Betriebe aus, die entweder fehlende Informationen auf der Betriebs- $(17,23 \%)$ oder der Individualebene (3,79\%) vorweisen. Somit besteht unser finales Sample aus 93 Betrieben $^{8}$ und 5072 Beschäftigten.

\subsection{Variablen}

Als abhängige Variable dient in dieser Untersuchung der logarithmierte Bruttostundenlohn der befragten Personen in Euro, in dem Überstunden und Transferzahlungen mitberücksichtigt werden. ${ }^{9}$ Weiterhin unterscheiden wir zwischen männlichen und weiblichen Beschäftigten und verwenden Männer als Referenzkategorie. Der durchschnittliche Stundenlohn von Männern liegt bei $28 €$ und der von Frauen bei $20 €$. Als Grundlage zur Differenzierung des Qualifikationsniveaus wird die CASMIN (Comparative Analysis of Social Mobility in Industrial Nations)-Klassifikation verwendet. Wir differenzieren zwischen Beschäftigten mit höherem und geringerem Qualifikationsniveau. Beschäftigte, die einen Hoch- oder Fachhochschulabschluss besitzen, zählen zu der Gruppe der höher qualifizierten. Als geringer qualifiziert gelten Beschäftigte mit ,keinem Abschluss“, „Hauptschulabschluss ohne/mit beruflicher Ausbildung“, „mittlerer Reife ohne/mit beruflicher Ausbildung“ oder „Fachhochschulreife/Abitur ohne/mit beruflicher Ausbildung". Die deskriptive Betrachtung zeigt, dass Frauen mit hohem Qualifikationsniveau durchschnittlich $25 €$ die Stunde verdienen, wohingegen ihre männlichen Kollegen $37 €$ die Stunde erhalten. Der durchschnittliche Stundenlohn von Männern mit geringerem Qualifikationsniveau liegt bei $21 €$ und der von Frauen bei $18 €$.

Auf der Betriebsebene liegt ein besonderer Fokus auf Gleichstellungsmaßnahmen, den Mentoringprogrammen und den betrieblichen Frauenquoten sowie den vorherrschenden Geschlechterideologien. Die Personalverantwortlichen wurden gebeten anzugeben, welche Maßnahmen sie zur Förderung der Chancengleichheit von Frauen und Männern im Betrieb anbieten. Sie konnten aus einer Liste von möglichen Maßnahmen wählen (Mehrfachnennungen möglich). Die beiden häufigsten Maßnahmen sind (1) Förderung des weiblichen Nachwuchses durch gezielte Karriereplanung, Mentoringprogramme für Frauen, Netzwerkgruppen für Frauen und (2) gezielte Förderung des weiblichen Nachwuchses durch bevorzugte Stellenbesetzung mit Frauen/Frauenquoten. ${ }^{10}$ Von den 93 Betrieben in unserem Sample haben

\footnotetext{
8 Von den 93 Betrieben sind 16 ostdeutsche Betriebe mit 819 Beschäftigten. Daher reicht die Anzahl nicht aus, um die Analysen getrennt für Ost- und Westdeutschland zu berechnen. Allerdings zeigt die Aufnahme eines Interaktionsterms in die Regressionsmodelle keine zentralen Veränderungen der nachfolgenden Ergebnisse.

${ }^{9}$ Durch das Logarithmieren des Bruttostundenlohns wird eine Angleichung an eine Normalverteilung vorgenommen. Darüber hinaus können die Koeffizienten somit als prozentuale Veränderungen interpretiert werden.

${ }^{10}$ Somit ist nicht auszuschließen, dass die bevorzugte Stellenbesetzung nur für weibliche Beschäftigte innerhalb des Betriebs gültig ist oder auch bei Neueinstellungen. Folglich kann die betriebliche Frauenquote nicht nur mit einer höheren Beförderungswahrscheinlichkeit von Kandidatinnen innerhalb der Belegschaft assoziiert sein, sondern auch mit der Einstellungswahrscheinlichkeit von neu rekrutierten Frauen.
} 
Tab. 1 Deskriptive Informationen zu den Betriebsmerkmalen $(N=93)$

\begin{tabular}{lllc}
\hline & Mittelwert & SD & Min./Max \\
\hline $\begin{array}{l}\text { Gleichstellungsmaßnahmen } \\
\text { Mentoringprogramme }\end{array}$ & 0,44 & 0,50 & $0 / 1$ \\
$\begin{array}{l}\text { Betriebliche Frauenquote } \\
\text { Geschlechterideologien }\end{array}$ & 0,25 & 0,44 & $0 / 1$ \\
$\begin{array}{l}\text { Durchschnittliche Zustimmung: ein Kleinkind wird darunter } \\
\text { leiden, wenn seine Mutter berufstätig ist }\end{array}$ & 3,01 & 0,46 & $2,15 / 3,92$ \\
$\begin{array}{l}\text { Standardabweichung: ein Kleinkind wird darunter leiden, } \\
\text { wenn seine Mutter berufstätig ist }\end{array}$ & 1,31 & 0,10 & $1,07 / 1,62$ \\
$\begin{array}{l}\text { Durchschnittliche Zustimmung: der Mann und die Frau soll- } \\
\text { ten beide zum Haushaltseinkommen beitragen }\end{array}$ & 3,89 & 0,33 & $3,31 / 4,81$ \\
$\begin{array}{l}\text { Standardabweichung: der Mann und die Frau sollten beide } \\
\text { zum Haushaltseinkommen beitragen }\end{array}$ & 1,05 & 0,13 & $0,46 / 1,31$ \\
\hline
\end{tabular}

$23(24,7 \%)$ eine betriebliche Frauenquote implementiert und 70 (75,3\%) nicht. Mentoringprogramme wurden demgegenüber in 40 (43\%) Betrieben implementiert, wohingegen 53 (57\%) Betriebe diese Maßnahme nicht implementiert haben. ${ }^{11}$

Um die vorherrschenden Dimensionen der Geschlechterideologien abzubilden, wurden Informationen auf der Individualebene aggregiert und jedem Betrieb wurde ein individueller Wert zugeordnet, der widerspiegelt, inwieweit die dort angestellten Beschäftigten den jeweiligen Dimensionen der Geschlechterideologien zustimmen. Als Grundlage dienen die Zustimmungen der Beschäftigten hinsichtlich der Aussagen (1) „Ein Kleinkind wird darunter leiden, wenn seine Mutter berufstätig ist“ und (2) „Der Mann und die Frau sollten beide zum Haushaltseinkommen beitragen“.12 Die Befragten konnten auf einer Skala von „, = Trifft voll und ganz zu“ bis , $5=$ Trifft überhaupt nicht zu“ ihre Zustimmung zu der jeweiligen Aussage bewerten. Die Skala der letzten Frage wurde umgedreht, sodass einheitlich hohe Werte mit niedriger Zustimmung und somit egalitären Ansichten einhergehen. Die Verteilung der durchschnittlichen Zustimmung innerhalb der Betriebe zu diesen beiden Items zeigt, dass es sowohl Betriebe gibt, in denen eher traditionelle Einstellungen überwiegen und Betriebe, in denen eher egalitäre Ansichten vorherrschen (Anhang, Abb. 4). Außerdem besteht eine größere Streuung zwischen den durchschnittlichen Zustimmungen für Item 1 (Kleinkinder werden darunter leiden) als für Item 2 (Mann und Frau sollten zum HH beitragen). Insgesamt ist zu erkennen, dass die durchschnittliche Zustimmung über alle Betriebe hinweg traditioneller für Item 1 (Kleinkind wird unter berufstätiger Mutter leiden) ausfällt. Um gleichzeitig die Varianz der Zustimmungslevel zu beiden Items innerhalb der Belegschaft abzubilden, wurde die Standardabweichung auf der Individualebene für jeden Betrieb aggregiert und in die Analysen aufgenommen (Tab. 1).

Es werden einkommensrelevante Merkmale, wie demografische Merkmale, Humankapitalausstattung, Beschäftigungsmerkmale und beruflicher Rang, kontrolliert

\footnotetext{
11 Damit liegt der Anteil höher als bei der Implementierung dieser beiden Maßnahmen mit dem Ziel, den Frauenanteil in Führungspositionen zu erhöhen (Frodermann et al. 2018).

12 Die Korrelation dieser beiden Items beträgt 0,25 .
} 


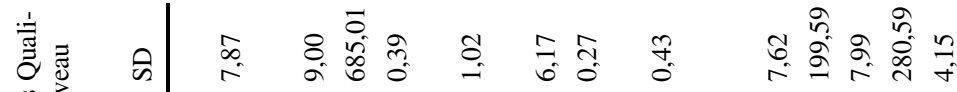

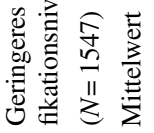

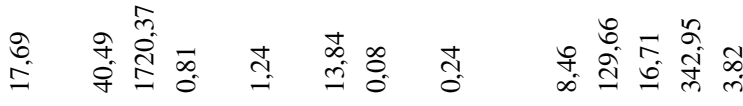

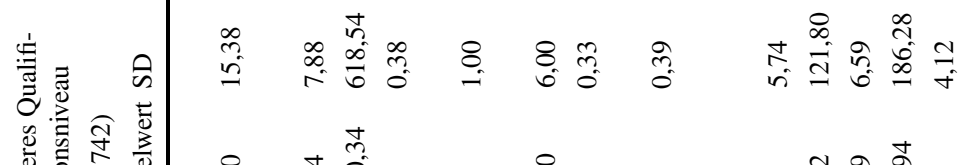

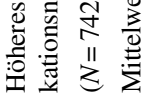
भु)

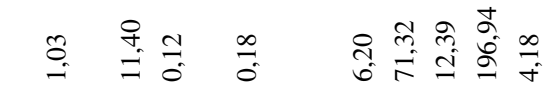

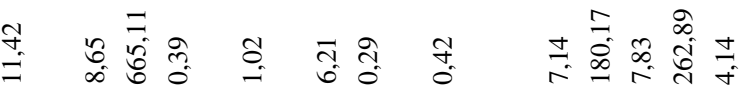

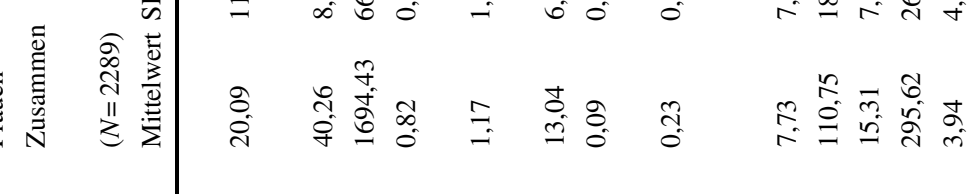
咅芩 की

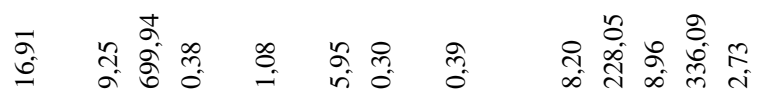
造

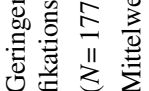
a

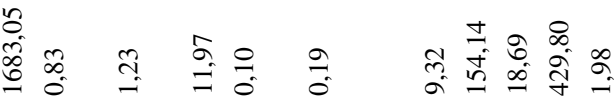
䇂芩 क

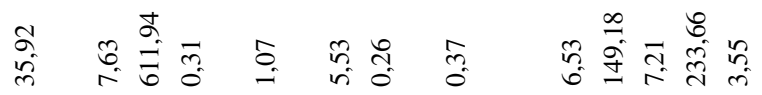

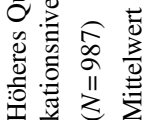

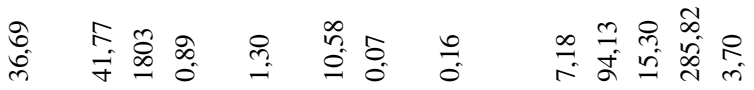

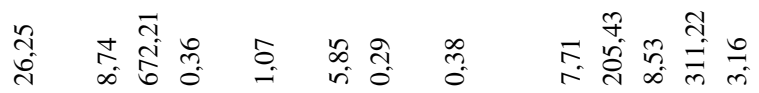
可 o.

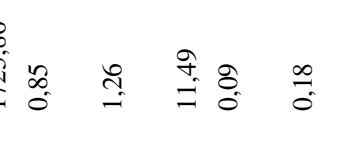

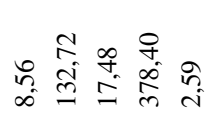
(1) 


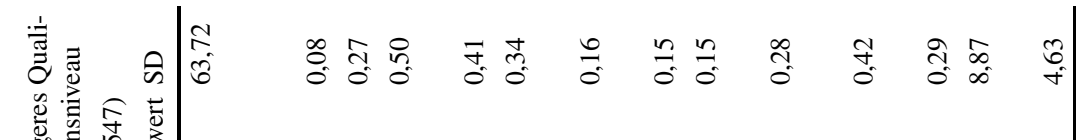

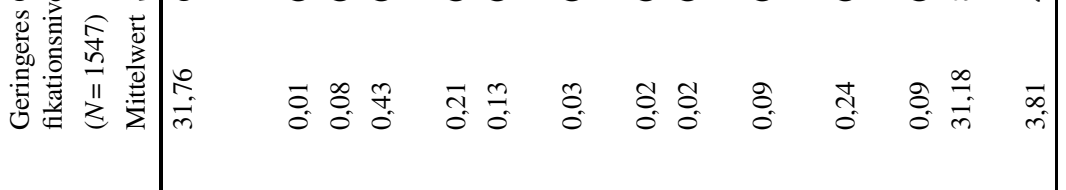

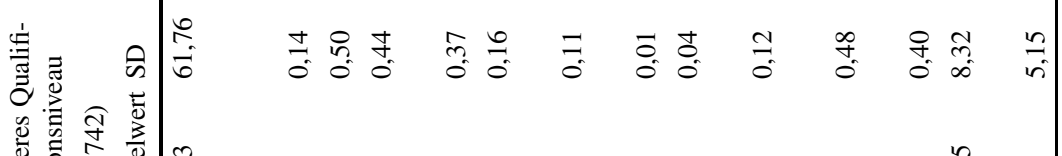

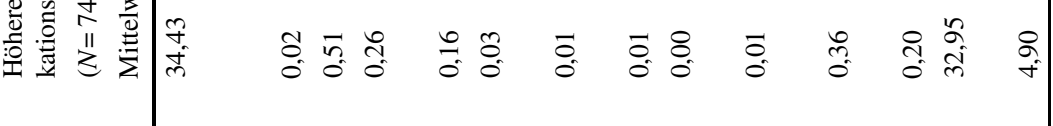

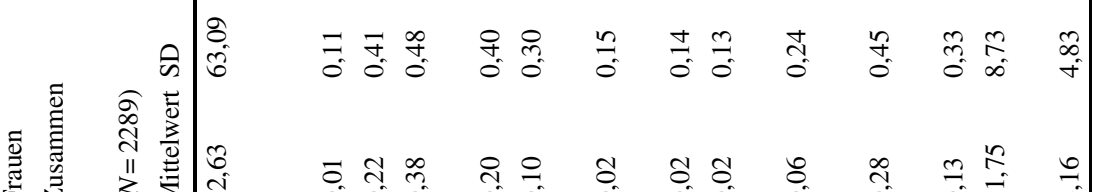

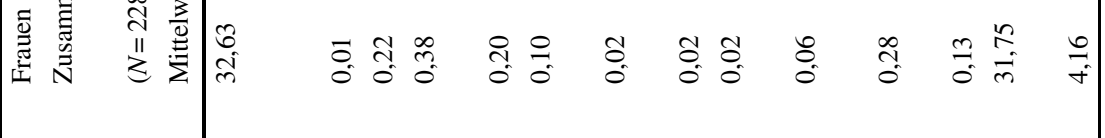

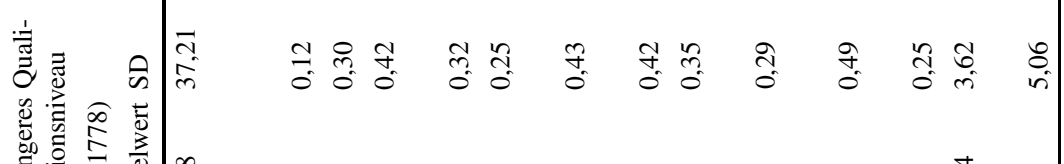

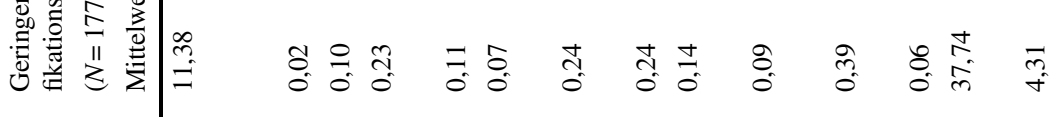

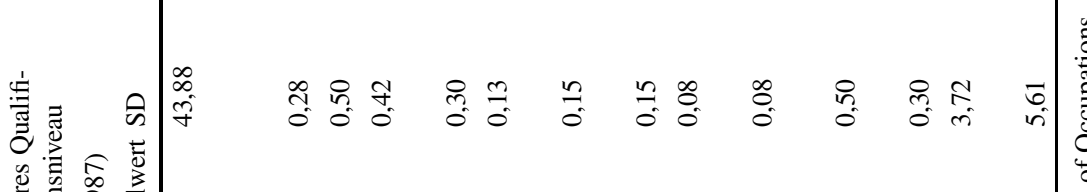

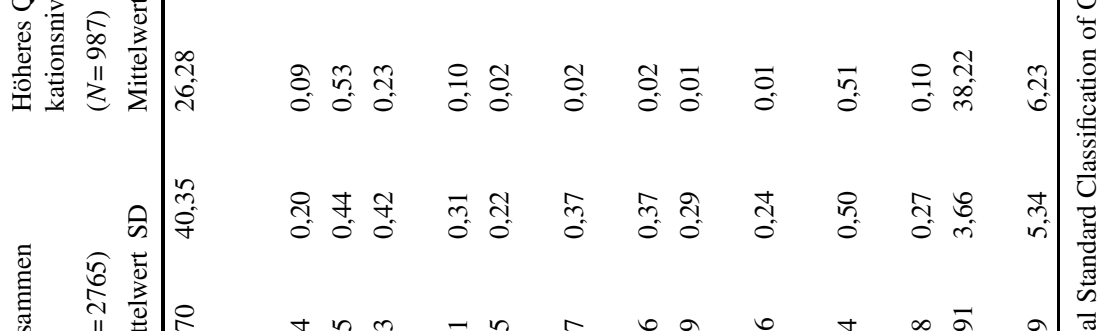

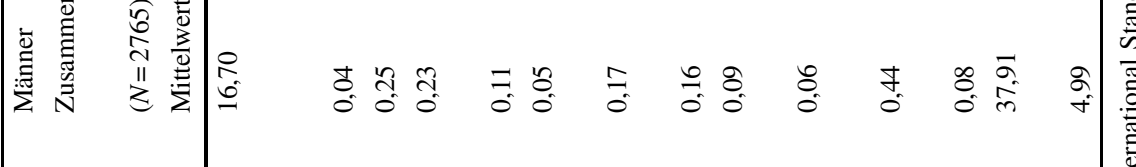

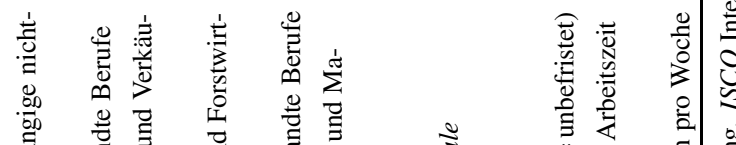

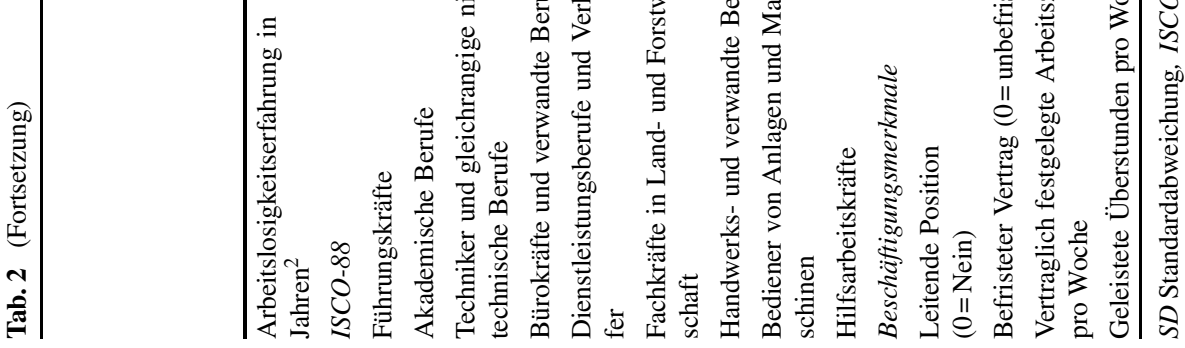


(Ganzeboom et al. 1992; Budig und England 2001; Gangl 2006; Giesecke und Verwiebe 2016) (Tab. 2).

Alter, Alter ${ }^{213}$ sowie Alter des jüngsten Kindes ${ }^{14}$ werden in Jahren gemessen. Partner $(0=$ kein Partner), Kinder $(0=$ keine Kinder $)$, Migrationshintergrund $(0=$ kein Migrationshintergrund) und Pflege von Angehörigen ( $0=$ keine Pflege) sind dichotome Variablen. Die Kinderanzahl wird als kategoriale Variable aufgenommen und unterscheidet zwischen keine Kinder, ein Kind, zwei Kinder und mehr als drei Kinder. Die Betriebszugehörigkeit, Arbeitsmarkterfahrung sowie Arbeitslosigkeitserfahrung in Jahren sowie der quadrierte Term, um nichtlineare Zusammenhänge zu kontrollieren, werden auf Grundlage der zugespielten administrativen Daten ermittelt, die taggenaue Informationen enthalten. Um wichtige Beschäftigungsmerkmale abzubilden, kontrollieren wir für die vertraglich festgelegten Arbeitsstunden, wöchentlich geleistete Überstunden, befristete Beschäftigung ( $0=$ unbefristet $)$ und leitende Position im Betrieb $(0=$ keine Leitungsfunktion). Um die berufliche Stellung der Beschäftigten abzubilden, wurde die einstellige ISCO-88-Klassifikation (International Standard Classification of Occupations) auf Basis der administrativen Daten verwendet. Der einstellige ISCO beinhaltet neun Kategorien, wobei hohe Werte einen niedrigeren beruflichen Rang darstellen. Da die höchste Kategorie (Führungskräfte) schwach besetzt ist, dienen in den Analysen akademische Berufe (Kategorie 2) als Referenzkategorie.

\subsection{Methode}

Aufgrund der hierarchischen Datenstruktur des verwendeten Datensatzes ist es notwendig, geeignete Methoden anzuwenden, die diese genestete Struktur berücksichtigen (Hinz 2009; Acock 2016). Genestete Daten liegen vor, wenn Individuen oder Beobachtungen in bestimmten Gruppen geclustert sind oder mehrere Beobachtungen für ein Individuum über die Zeit hinweg vorhanden sind (Acock 2016). Beschäftigte aus demselben Betrieb sind spezifischen Betriebsmerkmalen ausgesetzt, wie den implementierten Gleichstellungsmaßnahmen, dominierenden Geschlechterideologien und der demografischen Zusammensetzung, und sich somit ähnlicher als Beschäftigte aus anderen Betrieben. Die Annahme, dass alle Beobachtungen unabhängig voneinander sind, die Voraussetzung für OLS-Regressionsmodelle, ist somit verletzt. Als Resultat würden OLS-Schätzer die Standardfehler zu gering schätzen und es bestünde ein erhöhtes Risiko, fälschlicherweise signifikante Ergebnisse zu produzieren (Hox 2010). Im Einklang mit der Datenstruktur wurden Regressionsanalysen mit organisationsbezogenen Fixed Effects berechnet. Ein weiterer Vorteil von Fixed Effect-Modellen besteht darin, dass für unbeobachtete Heterogenität auf der höheren Ebene kontrolliert wird, selbst wenn diese mit dem Fehlerterm korreliert ist (Rabe-Hesketh und Skrondal 2012). Weiterhin berücksichtigen Fixed Effect-Modelle den besonderen Vorzug von hierarchischen Daten die Möglichkeit des WithinVergleichs, sodass durch diese Spezifikation Ungleichheiten zwischen Beschäftigten

\footnotetext{
13 Das Alter wird als quadrierter Term in die Analysen aufgenommen, um den nicht linearen Zusammenhang zwischen Einkommen und Alter abzubilden.

14 Personen, die keine Kinder haben, wurde der Mittelwert zugewiesen.
} 
innerhalb desselben Betriebes analysiert werden können. Somit können beispielsweise Selektionsprozesse von Männern und Frauen in unterschiedliche Betriebe berücksichtigt werden. Allerdings ist es in Fixed Effect-Regressionsmodellen nicht möglich, den Haupteffekt von Betriebsmerkmalen zu schätzen. Da unser Interesse auf dem Zusammenspiel der Betriebsmerkmale (Mentoringprogramme, betriebliche Frauenquote, Geschlechterideologien) mit dem Geschlecht liegt, stellt diese Einschränkung jedoch keine Limitation dar. Wir nehmen die Betriebsmerkmale als Interaktionsterm mit dem Geschlecht in unsere Modelle auf (Rabe-Hesketh und Skrondal 2012):

$\ln \left(\right.$ Stundenlohn $\left._{i b}\right)=\alpha_{0}+\alpha_{1}$ Geschlecht $_{i b}+\alpha_{2}\left(\right.$ Geschlecht $\left._{i b} * 0_{b}\right)+\delta_{1} Z_{i b}+u_{b}+E_{i b}$

Der Einkommensunterschied zwischen dem logarithmierten Stundenlohn von Männern und Frauen $\alpha_{1}$ wird basierend auf den individuellen Einkommen eines jeden Beschäftigten $i$ in Betrieb $b$ geschätzt. Alle weiteren Variablen, die individuelle Charakteristika beschreiben, wie beispielsweise das Alter oder die Arbeitsmarkterfahrung, werden durch den Vektor $Z_{i b}$ abgebildet. Schließlich repräsentiert $u_{b}{ }^{15}$ den unbeobachteten Betriebsebenen Fixed Effect-Fehlerterm und $E_{i b}$ den individuell spezifischen Fehlerterm. Die Betriebsmerkmale werden durch $0_{b}$ abgebildet, sodass Geschlecht $_{i b} * 0_{b}$ die Interaktionsterme darstellen. Die Interaktionsterme geben an, wie viel Frauen im Vergleich zu Männern in Betrieben verdienen, die beispielsweise keine Mentoringprogramme implementiert haben. In allen Modellen schätzen wir geclusterte robuste Standardfehler (Abadie et al. 2017).

\section{Ergebnisse}

Tabelle 3 zeigt Fixed Effect-Regressionsmodelle, die den logarithmierten Stundenlohn für alle Beschäftigten und separat für Beschäftigte mit höherem und geringerem Qualifikationsniveau unter Berücksichtigung des Geschlechts schätzen. Die Kontrollvariablen werden schrittweise in die Modelle aufgenommen. Die unbereinigte Lohnlücke zwischen Frauen und Männern mit hohem Qualifikationsniveau beträgt 17,4\%, wohingegen die Einkommen von Frauen mit geringerem Qualifikationsniveau 10,8\% unter dem ihrer männlichen Kollegen liegen. Diese Lohnlücke sinkt allerdings unter Berücksichtigung von produktivitätsrelevanten Merkmalen auf 7,8\% für Beschäftigte mit hohem Qualifikationsniveau. Demgegenüber zeigt sich, dass bei Beschäftigten mit geringerem Qualifikationsniveau die Lohnlücke zwischen Frauen und Männern mit 11,7\% höher ausfällt als die unbereinigte Lohnlücke. ${ }^{16}$

\footnotetext{
15 Wobei der Fehlerterm in Fixed Effect-Modellen auf der Betriebsebene einen Wert von 0 annimmt.

16 Dies ist auf die Berücksichtigung des beruflichen Rangs der Beschäftigten zurückzuführen (Vergleich Modell 3b zu 3c in Tab. 3).
} 


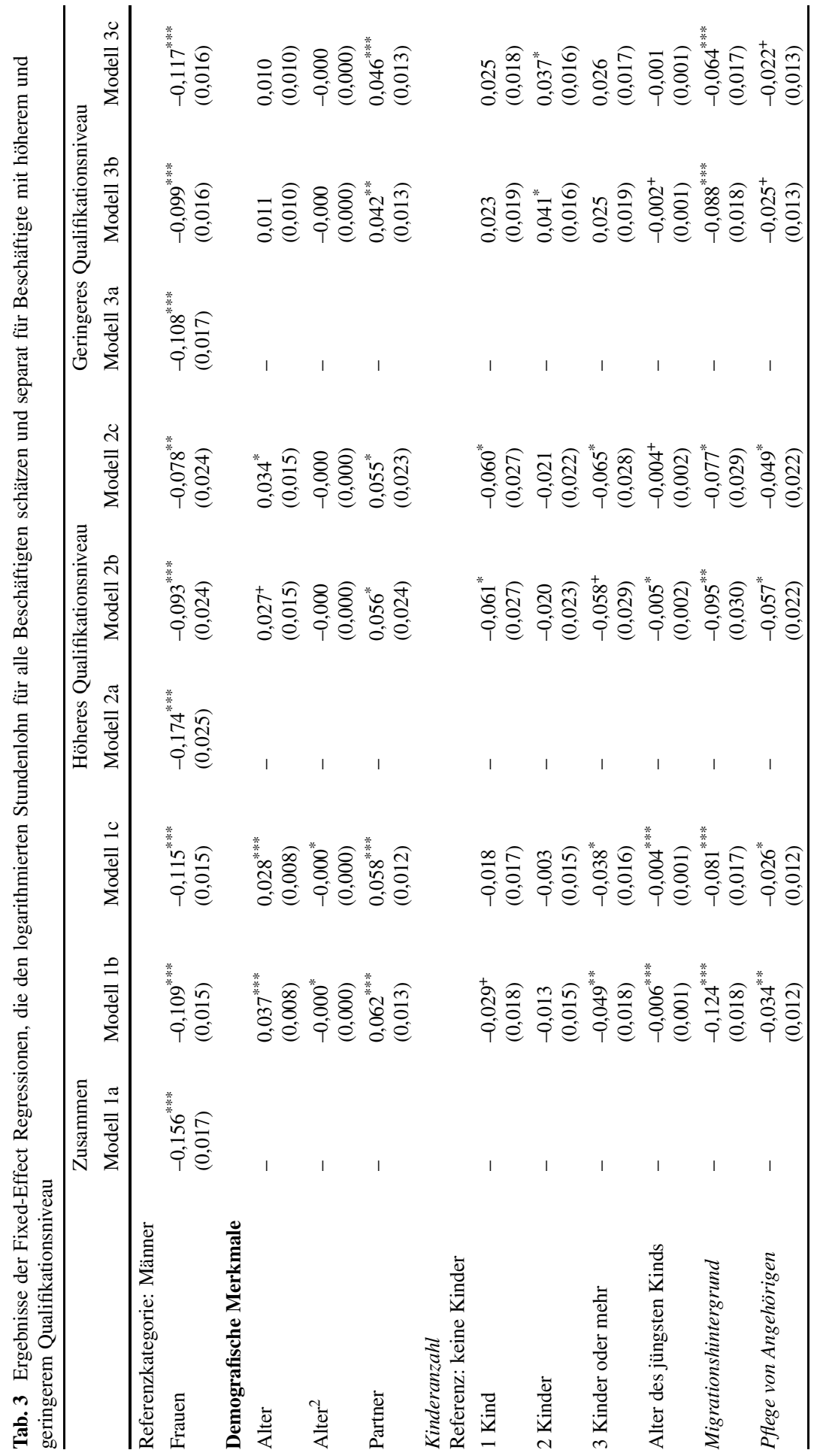




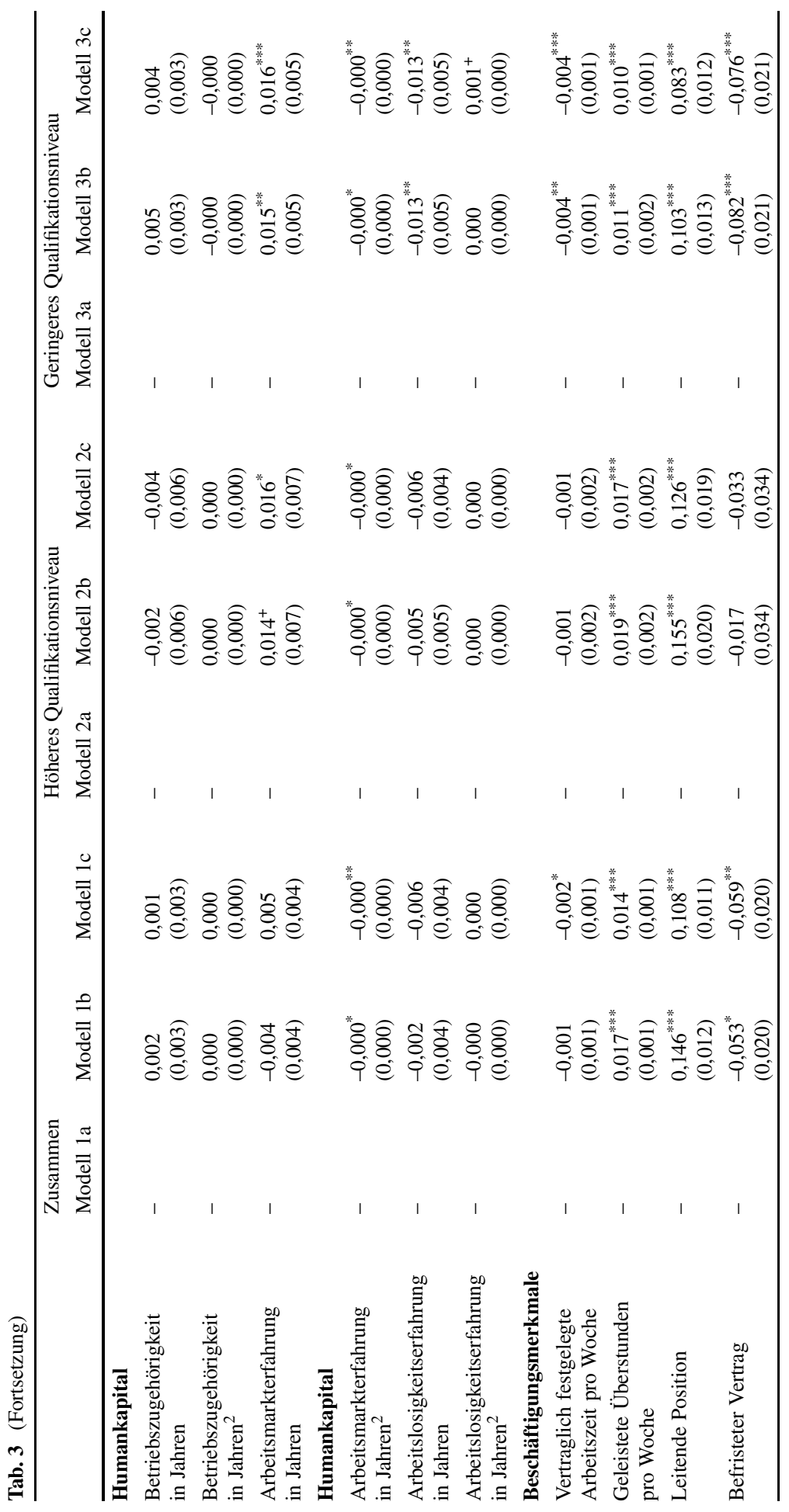




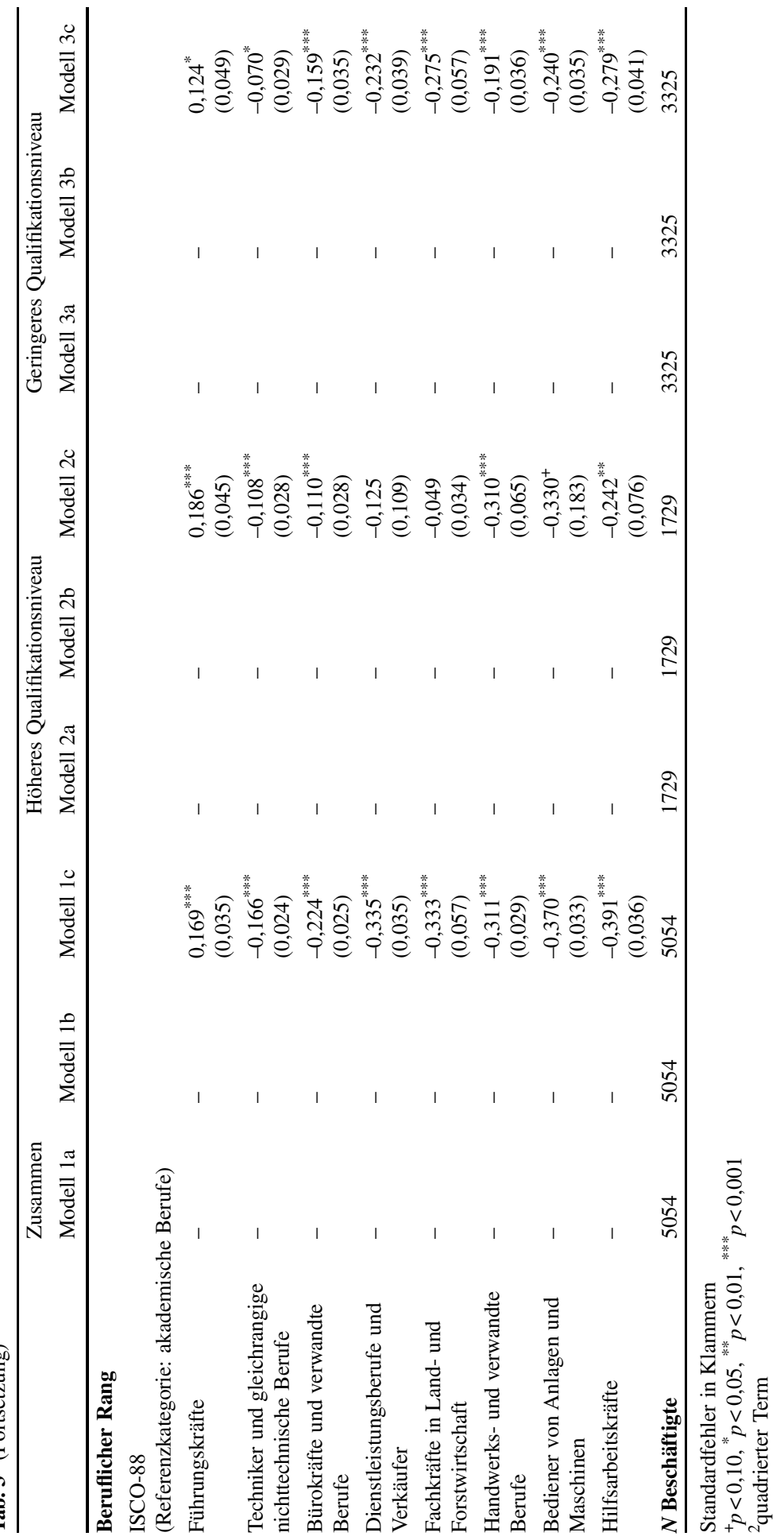




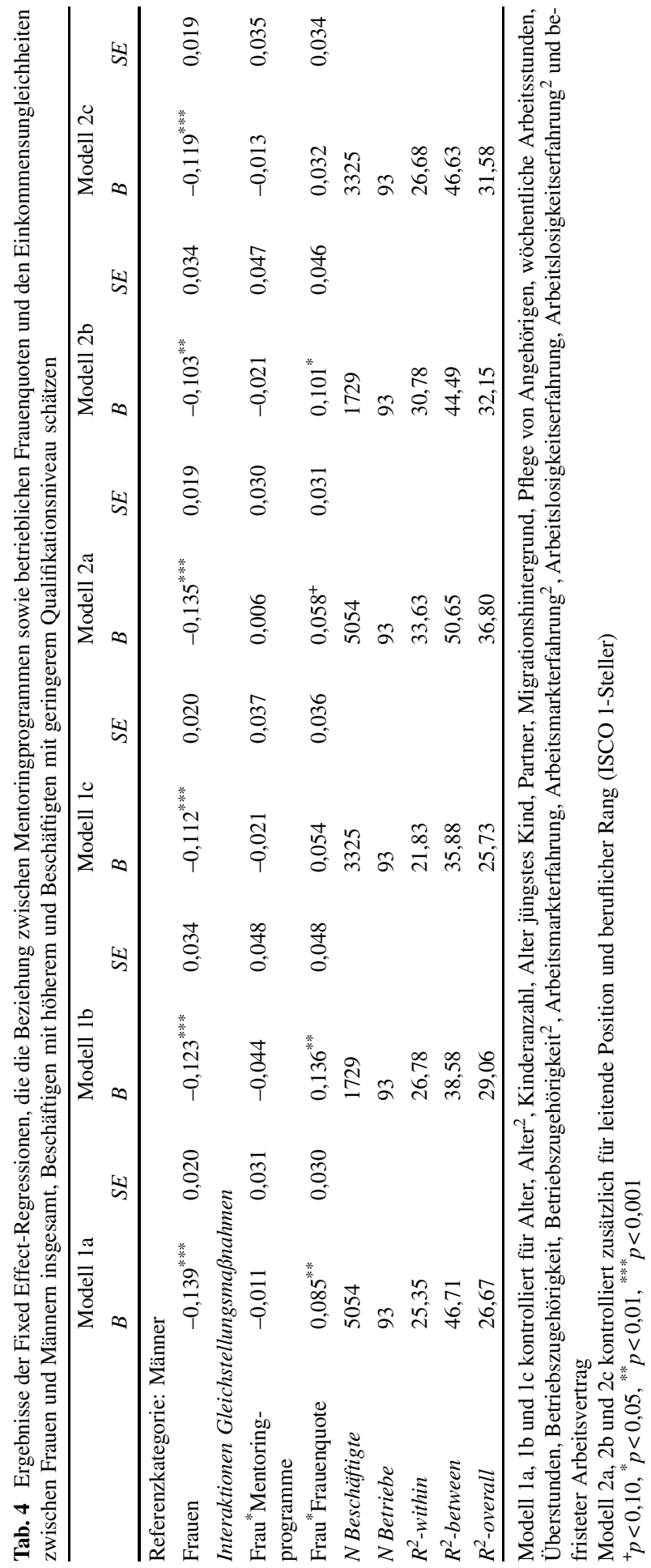




\subsection{Gleichstellungsmaßnahmen}

Die Hypothesen 1a-1d werden in Tab. 4 anhand von Fixed Effect-Regressionsmodellen getestet, die den Zusammenhang von Mentoringprogrammen sowie der betrieblichen Frauenquote mit den geschlechtsspezifischen Einkommensungleichheiten abbilden. Um zu analysieren, inwieweit die Gleichstellungsmaßnahmen über die berufliche Stellung hinaus mit Einkommensunterschieden assoziiert sind, wurden die Modelle zuerst ohne Kontrollvariable für den beruflichen Rang berechnet (Modell 1a, 1b und 1c) und anschließend unter Berücksichtigung des beruflichen Rangs (Modell 2a, 2b und 2c). In beiden Schritten wird zwischen dem gesamten Sample (a), Beschäftigten mit höherem Qualifikationsniveau (b) und Beschäftigten mit geringerem Qualifikationsniveau (c) unterschieden. Dieses Vorgehen ermöglicht die Untersuchung der unterschiedlichen Mechanismen, die über die Gleichstellungsmaßnahmen gefördert werden: die Selektion in bestimmte Berufe sowie Positionen in der Betriebshierarchie und der Abbau von Stereotypisierungsprozessen.

Die Ergebnisse in Tab. 4 zeigen, dass Mentoringprogramme für keines der betrachteten Samples einen signifikanten Zusammenhang mit den geschlechtsspezifischen Einkommensungleichheiten im Betrieb besitzen; weder vor noch nach der Kontrolle für den beruflichen Rang der Beschäftigten. Demnach scheint die Implementation von Mentoringprogrammen weder mit einer Selektion von Frauen in bestimmte Berufe und Positionen assoziiert zu sein noch mit dem Abbau von Stereotypisierungsprozessen. Hypothese 1a kann anhand der vorliegenden Ergebnisse weder für Beschäftigte mit hohem noch für Beschäftigte mit geringem Qualifikationsniveau bestätigt werden.

Demgegenüber besteht ein positiver Zusammenhang zwischen der betrieblichen Frauenquote und geschlechtsspezifischen Einkommensungleichheiten innerhalb von Betrieben. Dieser Zusammenhang lässt sich allerdings nur für die Gruppe der Beschäftigten mit höherem Qualifikationsniveau vorfinden. Dies mag zum einen auf einen höheren Lohnzuwachs von Frauen in Betrieben mit Frauenquote im Vergleich zu Männern zurückzuführen sein oder auf eine Lohnangleichung der Einkommen von Männern an das von Frauen oder einer Kombination aus beidem. Demgegenüber sind die geschlechtsspezifischen Einkommensungleichheiten zwischen Beschäftigten mit geringerem Qualifikationsniveau nicht mit der Implementierung einer Frauenquote assoziiert. Die geschlechtsspezifischen Einkommensungleichheiten zwischen Beschäftigten mit hohem Qualifikationsniveau sind in Betrieben mit Frauenquote $14,6 \%{ }^{17}$ geringer als in Betrieben ohne Frauenquote (Tab. 4, Modell 1b). Unter Berücksichtigung der beruflichen Stellung der Beschäftigten bleibt der positive Zusammenhang zwischen der Frauenquote und den geschlechtsspezifischen Einkommensungleichheiten bestehen, wonach die Frauenquote über die Selektion in bestimmte Berufe hinaus mit geringeren bereinigten Einkommensungleichheiten assoziiert ist. Die Lohnlücke zwischen Frauen und Männern mit hohem Qualifikationsniveau ist in Betrieben mit Frauenquote $10,6 \%^{18}$ geringer als in Betrieben ohne

$17\left(e^{0,136}-1\right) * 100 \sim 14,6 \%$.

$18\left(e^{0,101}-1\right) * 100 \sim 10,6 \%$. 


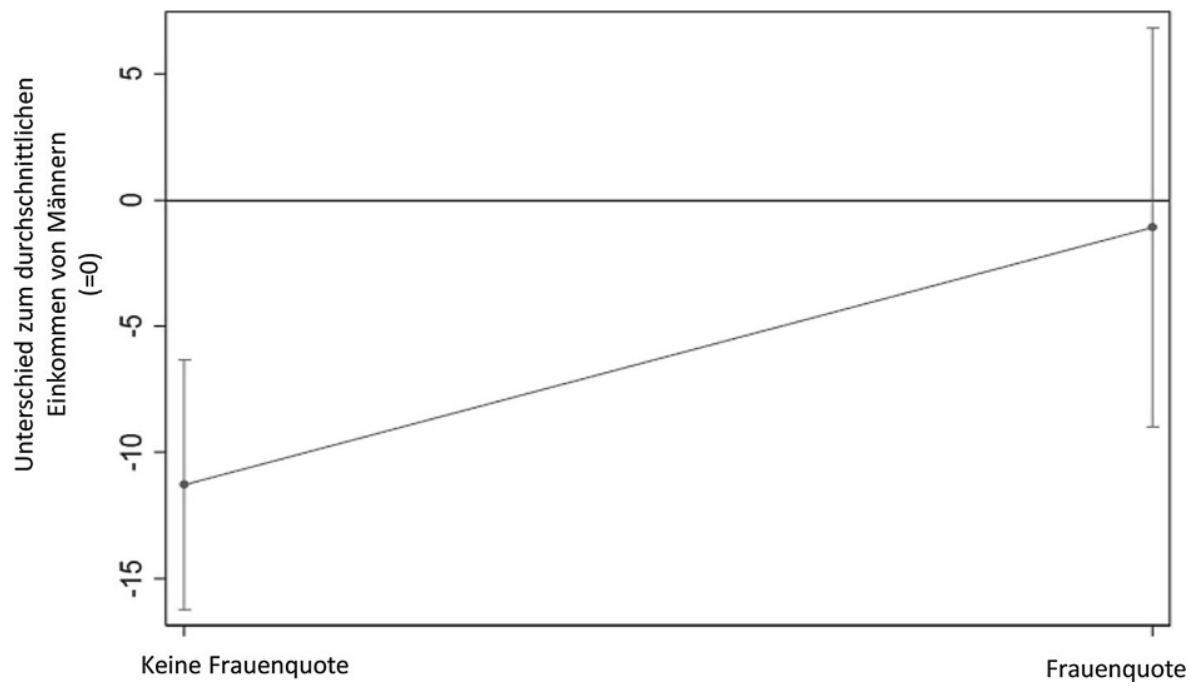

Abb. 1 Vorhergesagte Einkommensbenachteiligungen von hochqualifizierten Frauen in Betrieben mit und ohne betrieblicher Frauenquote (Abbildung wurde anhand der Fixed Effect-Regression aus Tab. 4, Modell $2 \mathrm{~b}$ berechnet. Bereich $=95 \%$ Konfidenzintervalle)

Frauenquote (Tab. 2, Modell 2b). Abbildung 1 veranschaulicht den Zusammenhang zwischen der betrieblichen Frauenquote und den Einkommensdifferenzen von Beschäftigten mit höherem Qualifikationsniveau innerhalb von Betrieben mit und ohne Frauenquote.

In Abb. 1 wird der Unterschied zwischen dem Einkommen von Frauen im Vergleich zum Einkommen ihrer männlichen Kollegen in Betrieben mit und ohne Frauenquote abgebildet. Es ist zu erkennen, dass in Betrieben mit einer Frauenquote keine signifikanten Einkommensdifferenzen zwischen Frauen und Männern innerhalb des Betriebs bestehen, da die Konfidenzintervalle die Referenzlinie überschneiden. Demgegenüber lässt sich eine substanzielle Lohnlücke in Betrieben ohne Frauenquote vorfinden. Frauen mit höherem Qualifikationsniveau verdienen in Betrieben ohne Frauenquote über $10 \%$ pro Stunde weniger als ihre männlichen Kollegen. Folglich kann Hypothese 1b, die annimmt, dass eine positive Assoziation zwischen der betrieblichen Frauenquote und geringeren geschlechtsspezifischen Einkommensungleichheiten besteht, nur für Beschäftigte mit hohem Qualifikationsniveau bestätigt werden. In Hypothese 1c wurde angenommen, dass der Zusammenhang für betriebliche Frauenquoten stärker im Vergleich zu Mentoringprogrammen ausfällt. Hypothese 1c findet somit Bestätigung, zumindest für Beschäftigte mit hohem Qualifikationsniveau, da nur betriebliche Frauenquoten mit geringeren Einkommensunterschieden assoziiert sind. Weiterhin findet die Annahme, dass nicht nur die Selektion in bestimmte Berufe, sondern auch bereinigte Einkommensunterschiede geringer ausfallen, Bestätigung. 


\subsection{Gleichstellungsmaßnahmen und Geschlechterideologien}

Im nächsten Schritt wird geprüft, inwieweit die zuvor dargestellten Zusammenhänge der Gleichstellungsmaßnahmen mit den geschlechtsspezifischen Einkommensungleichheiten durch die vorherrschenden Geschlechterideologien im Betrieb moderiert werden. Dieser mögliche Zusammenhang wird über die Aufnahme von Dreifach-Interaktionen getestet. Die Tabellen 5 und 6 beinhalten die Ergebnisse für Beschäftigte mit höherem und geringerem Qualifikationsniveau.

Der Zusammenhang einer betrieblichen Frauenquote mit Einkommensungleichheiten zwischen Frauen und Männern mit höherem Qualifikationsniveau scheint durch die vorherrschende Einstellung hinsichtlich der Berufstätigkeit von Müttern moderiert zu werden (Tab. 5, Modell 1b). Somit besitzt die Implementierung einer Frauenquote nicht in jedem betrieblichen Kontext einen positiven Zusammenhang mit der Einkommensangleichung, sondern er wird durch die vorherrschenden Geschlechterideologien in der Belegschaft mitbestimmt (Abb. 2).

Die geschlechtsspezifischen Einkommensungleichheiten sind in Betrieben ohne Frauenquote unabhängig von der vorherrschenden Geschlechterideologie vorzufinden. In Betrieben ohne Frauenquote und mit traditionellen Einstellungen in der Belegschaft beträgt die Einkommensdifferenz zwischen Frauen und Männern ca. $10 \%$ und in Betrieben ohne Frauenquote und mit egalitären Geschlechterideologien $12,7 \%$. Demgegenüber zeigt sich, dass die geschlechtsspezifischen Einkommensungleichheiten in Betrieben mit Frauenquote nur in Betrieben mit traditionellen Einstellungen in der Belegschaft vorzufinden sind. Frauen in Betrieben mit Frauenquote und traditionellen Ansichten in der Belegschaft verdienen 19,1\% weniger als ihre männlichen Kollegen im Betrieb. Dagegen bestehen keine signifikanten Einkommensunterschiede zwischen Frauen und Männern mit höherem Qualifikationsniveau in Betrieben mit Frauenquote und egalitären Einstellungen. Somit ist die Implementierung einer Frauenquote nicht per se mit geringeren Einkommensunterschieden zwischen Frauen und Männern assoziiert, sondern ist von der vorherrschenden Geschlechterideologie abhängig. Wie sich diese Zusammenhänge für die Einstellungen bezüglich der gemeinsamen Erwirtschaftung des Haushaltseinkommens von Frauen und Männern gestalten, veranschaulicht Abb. 3.

Es ist zu erkennen, dass in Betrieben, die eine Frauenquote implementiert haben, keine geschlechtsspezifischen Einkommensunterschiede zwischen Beschäftigten mit höherem Qualifikationsniveau bestehen, weder in traditionellen noch in egalitären Betriebskontexten. Demgegenüber bestehen in Betrieben ohne Frauenquote und mit traditionellen Ansichten über die Erwirtschaftung des Haushaltseinkommens keine signifikanten Einkommensunterschiede zwischen Frauen und Männern, jedoch aber in Betrieben mit egalitären Einstellungen. Der signifikante Interaktionsterm scheint sich aus der gegenteiligen Steigung in Abhängigkeit der Geschlechterideologie für Betriebe mit und ohne Frauenquote zu speisen.

Insgesamt bestehen keine geschlechtsspezifischen Einkommensunterschiede in Betrieben, die eine Frauenquote implementiert haben (Abb. 1). Allerdings verdienen Frauen in traditionellen Kontexten, gemessen an der Zustimmung zu der Aussage, dass Kleinkinder unter einer berufstätigen Mutter leiden werden, signifikant 


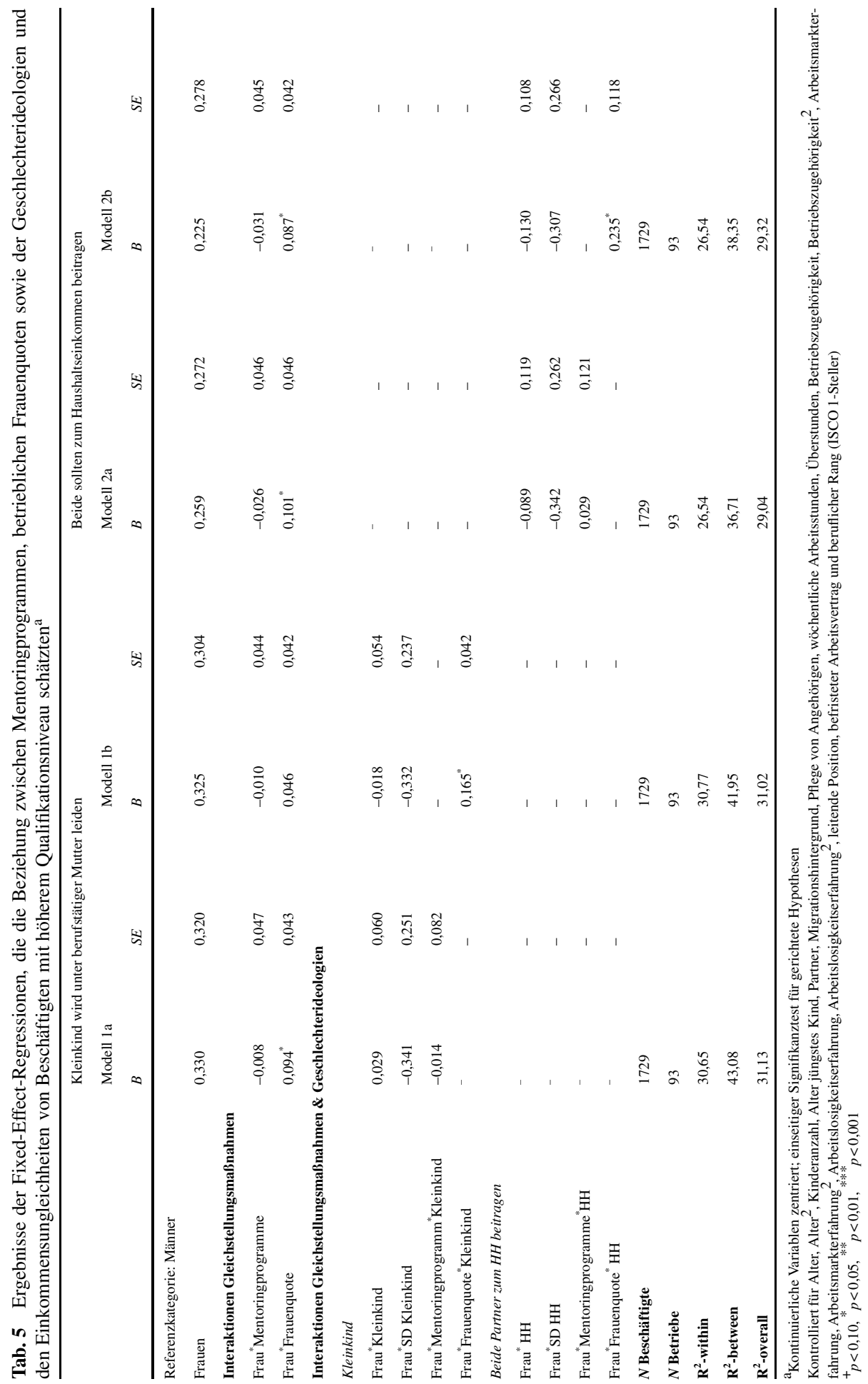




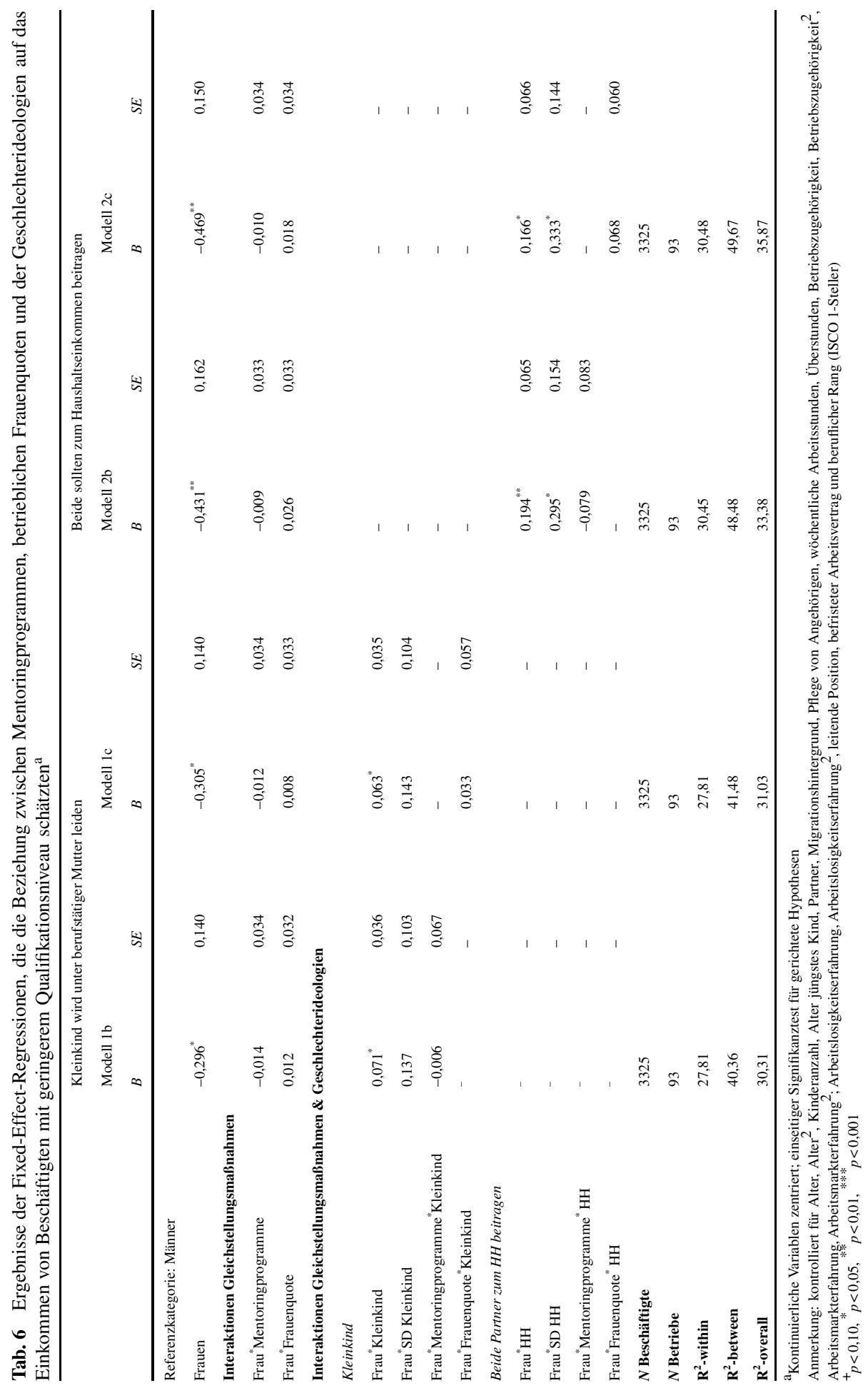




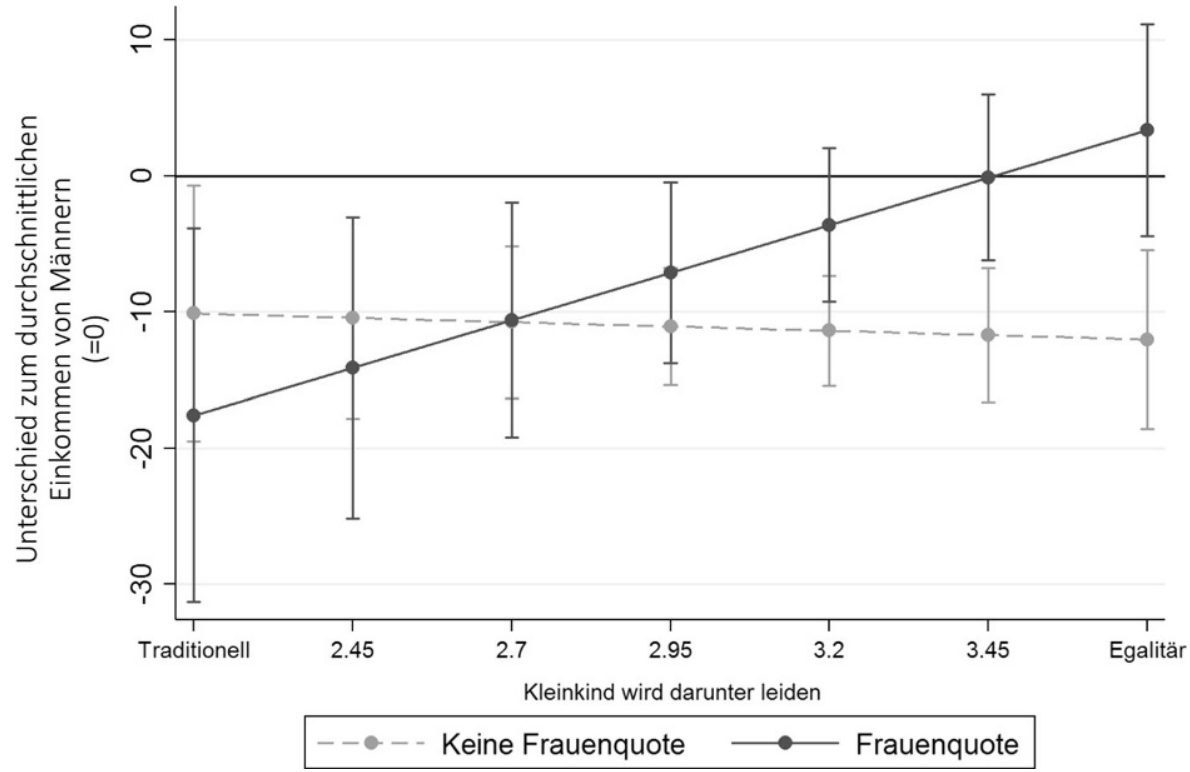

Abb. 2 Vorhergesagte Einkommensbenachteiligungen von hochqualifizierten Frauen in Betrieben mit und ohne betrieblicher Frauenquote in traditionellen und egalitären Betriebskontexten (Abbildung wurde anhand der Fixed Effect-Regression aus Tab. 5, Modell $1 \mathrm{~b}$ berechnet. Bereich $=90 \%$ Konfidenzintervalle)

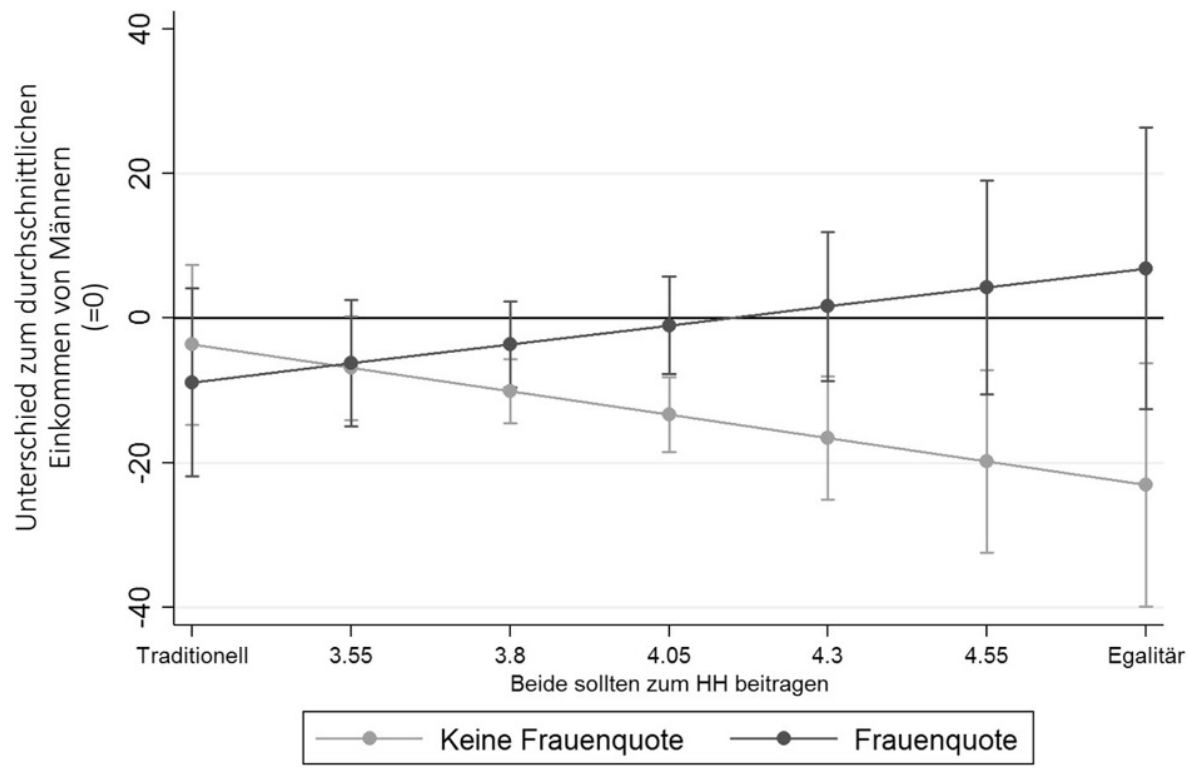

Abb. 3 Vorhergesagte Einkommensbenachteiligungen von hochqualifizierten Frauen in Betrieben mit und ohne betrieblicher Frauenquote in traditionellen und egalitären Betriebskontexten (Abbildung wurde anhand der Fixed Effect-Regression aus Tab. 5, Modell 2b berechnet. Bereich $=90 \%$ Konfidenzintervalle) 
weniger als ihre männlichen Kollegen. Demgegenüber bestehen keine geschlechtsspezifischen Einkommensungleichheiten in Betrieben mit Frauenquote in Abhängigkeit von der Einstellung bezüglich der gemeinsamen Erwirtschaftung des Haushaltseinkommens. Ob die vorherrschenden Geschlechterideologien den Zusammenhang zwischen betrieblichen Frauenquoten und Einkommensungleichheiten moderieren, scheint somit abhängig davon zu sein, welche Dimension der Geschlechterideologie betrachtet wird. Somit lassen sich teilweise Belege dafür finden, dass traditionelle Geschlechterideologien im Betrieb die Assoziation von Gleichstellungsmaßnahmen mit Einkommensungleichheiten zwischen Frauen und Männern mit höherem Qualifikationsniveau moderieren. Dagegen lässt sich nicht feststellen, dass Geschlechterideologien den Zusammenhang zwischen Gleichstellungsmaßnahmen und geschlechtsspezifischen Einkommensungleichheiten zwischen Beschäftigten mit geringerem Qualifikationsniveau beeinflussen. Somit scheinen Gleichstellungsmaßnahmen auch in egalitären betrieblichen Kontexten keinen Zusammenhang mit den Einkommensdifferenzen zwischen Beschäftigten mit geringerem Qualifikationsniveau aufzuweisen. Folglich kann Hypothese 2 nur für Beschäftigte mit höherem Qualifikationsniveau in Betrieben mit Frauenquote und traditionellen Einstellungen hinsichtlich der Berufstätigkeit von Müttern bestätigt werden.

Die Interaktionen der Geschlechterideologien mit dem Geschlecht zeigen, dass egalitäre Geschlechterideologien in der Belegschaft mit geringeren geschlechtsspezifischen Einkommensungleichheiten zwischen Frauen und Männern mit geringerem Qualifikationsniveau assoziiert sind. Demgegenüber besteht kein signifikanter Zusammenhang zwischen den Lohnlücken von Beschäftigten mit höherem Qualifikationsniveau und den vorherrschenden Geschlechterideologien. Demzufolge bestehen selbst in Betriebskontexten mit egalitären Einstellungen in der Belegschaft signifikante Einkommensunterschiede zwischen Frauen und Männern mit höherem Qualifikationsniveau. Dieser Befund deutet darauf hin, dass auch in egalitären Betrieben Barrieren für hochqualifizierte Frauen bestehen, die es ihnen erschweren, Einkommensparität mit ihren männlichen Kollegen zu erreichen.

\section{Diskussion und Fazit}

Trotz einer erhöhten Partizipation am Arbeitsmarkt und der Ein- oder Überholung von Männern hinsichtlich des Qualifikationsniveaus bestehen in Deutschland persistente Einkommensunterschiede sowie eine Unterrepräsentation von Frauen in Führungspositionen. Seit der Einführung des Allgemeinen Gleichbehandlungsgesetzes im Jahr 2006 besteht erhöhter Druck auf deutsche Betriebe, diesen Benachteiligungen entgegenzuwirken. Viele Betriebe haben daher Gleichstellungsmaßnahmen implementiert, um die Chancengleichheit von Frauen und Männern zu fördern. Empirische Analysen, inwieweit diese Maßnahmen tatsächlich zu geringeren Einkommensdifferenzen führen, sind allerdings rar (Huffman et al. 2017). Vor diesem Hintergrund lag das Ziel des vorliegenden Beitrags darin, eine differenzierte Untersuchung vorzunehmen, inwiefern Mentoringprogramme und freiwillige Frauenquoten mit geringeren geschlechtsspezifischen Einkommensunterschieden in Großbetrieben assoziiert sind. Weiterhin sollte analysiert werden, inwieweit diese Zusammenhän- 
ge durch das Qualifikationsniveau und die vorherrschenden Geschlechterideologien innerhalb der Belegschaft moderiert werden. Zudem wurde untersucht, inwiefern die geringeren Einkommensunterschiede in Betrieben mit Mentoringprogrammen oder Frauenquoten auf die bessere Positionierung von Frauen zurückgeführt werden können oder ob darüber hinaus Zusammenhänge mit bereinigten Einkommensunterschieden bestehen.

Unsere Ergebnisse zeigen, dass es sowohl Gleichstellungsmaßnahmen gibt, die mit geringeren geschlechtsspezifischen Einkommensungleichheiten assoziiert sind, als auch Maßnahmen, die keine Zusammenhänge vorweisen. So ist das Angebot von Mentoringprogrammen weder für Beschäftigte mit höherem noch für Beschäftigte mit geringerem Qualifikationsniveau mit verringerten Lohnlücken assoziiert. Dies könnte darauf zurückgeführt werden, dass Frauen zwar machtvolle Verbündete im Betrieb erhalten, diese Netzwerke allerdings nicht ausreichen, um die Barrieren und Hindernisse im Betrieb zu überwinden. Bisherige Forschung konnte zeigen, dass Frauen nur geringfügig von Mentoringprogrammen profitieren, da sich ihre Mentoren selten aktiv für ihr berufliches Fortkommen einsetzten (Ibarra et al. 2010). Weiterhin ist anzunehmen, dass die sozialen Netzwerke von Frauen zwar durch den zugewiesenen Mentor ausgebaut werden, möglicherweise aber nicht darüber hinaus. Somit lässt sich die in der Literatur bestehende Aussage, dass Mentoringprogramme dazu beitragen, geschlechtsspezifische Ungleichheiten abzubauen (Scandura 1992; Kurmeyer und Höppel 2017; Burke 1984; Fragenson 1988; Whitely et al. 1991) für die Einkommensungleichheiten in deutschen Großbetrieben nicht bestätigen.

Demgegenüber bestehen keine Einkommensunterschiede zwischen Frauen und Männern mit höherem Qualifikationsniveau in Betrieben mit einer freiwilligen Frauenquote, wohingegen hohe geschlechtsspezifische Einkommensungleichheiten in Betrieben ohne Frauenquote vorzufinden sind. Dieser Befund ist in Einklang mit der Forschung, die gezeigt hat, dass Frauenquoten für Betriebsvorstände geschlechtsspezifische Einkommensungleichheiten zwischen Vorstandsvorsitzenden abbauen (Bertrand et al. 2018; Maume et al. 2019). Durch den Vergleich der Lohnlücken mit und ohne Berücksichtigung der beruflichen Stellung von Beschäftigten wird weiterhin deutlich, dass sowohl die berufliche Geschlechtersegregation geringer ausfällt als auch die bereinigten Einkommensunterschiede. Demnach scheint die betriebliche Frauenquote die sozialen Schließungsprozesse, aufgrund derer Frauen einen erschwerten oder gar keinen Zugang zu gewissen Positionen in der Betriebshierarchie haben, abzuschwächen. Aufgrund der besseren Positionierung von Frauen und der daraus resultierenden Statusinkonsistenz können Wahrnehmungen und Bewertungen gegenüber Frauen als weniger kompetent und produktiv verändert werden. Die Einkommensunterschiede zwischen Beschäftigten mit geringerem Qualifikationsniveau weisen hingegen keinen signifikanten Zusammenhang mit einer betrieblichen Frauenquote auf. Die bessere Positionierung von Frauen am oberen Ende der Betriebshierarchie geht somit nicht mit geringeren Einkommensunterschieden von Frauen und Männern in unteren Statusgruppen einher. Folglich widersprechen unsere Ergebnisse vorheriger Forschung für Deutschland, die zeigt, dass Gleichstellungsmaßnahmen die Einkommensdifferenzen zwischen Frauen und Männern in den unteren zwei Dritteln der Einkommensverteilung verringern (Huffman et al. 2017). Allerdings wurde in dieser Untersuchung nicht zwischen einzelnen Gleich- 
stellungsmaßnahmen differenziert, sondern der generelle Einfluss des Vorhandenseins mindestens einer Gleichstellungsmaßnahme untersucht. Zudem wurden in den vorliegenden Analysen Einkommensungleichheiten innerhalb von Großbetrieben betrachtet, wohingegen das Sample von Huffman und Kollegen (2017) Betriebe ab 10 und mehr Beschäftigten umfasst.

Die variierenden Zusammenhänge von Mentoringprogrammen und betrieblichen Frauenquoten lassen sich vermutlich darauf zurückführen, dass sie unterschiedliche Ansätze verfolgen. Mentoringprogramme stellen ein Werkzeug zur Verfügung, um Forderungen besser durchzusetzen, Beziehungen zu machtvollen Akteuren im Betrieb aufzubauen und hierüber Benachteiligungen von weiblichen Beschäftigten aufzuheben. ${ }^{19}$ Demgegenüber besitzt eine betriebliche Frauenquote einen kürzeren Wirkungsweg, da Frauen der Zugang zu bestimmten Positionen direkt ermöglicht wird. Insgesamt legen die Ergebnisse nahe, dass nicht nur die differenzierte Betrachtung von Gleichstellungsmaßnahmen Aufschlüsse über die Assoziation mit geringeren Einkommensungleichheiten bieten kann, sondern auch die Unterscheidung zwischen Beschäftigten entlang des Qualifikationsniveaus. Denn um den persistenten Lohnlücken entgegenzuwirken, ist es notwendig, zu wissen, wo und wie Gleichstellungsmaßnahmen mit geringeren Einkommensbenachteiligungen einhergehen.

Darüber hinaus haben wir argumentiert, dass Gleichstellungsmaßnahmen nicht in jedem betrieblichen Kontext mit geringeren Lohnlücken assoziiert sind, sondern durch die vorherrschenden Geschlechterideologien innerhalb der Belegschaft moderiert werden können. Hiermit folgen wir der Forschung, die gezeigt hat, dass die Effektivität von Gleichstellungsmaßnahmen von den Einstellungen in der Belegschaft abhängig ist (Kalev et al. 2006; Dobbin et al. 2015; Kaiser et al. 2013). Unsere Ergebnisse belegen, dass eine freiwillige Frauenquote nicht per se mit Einkommensparität für Beschäftigte mit höherem Qualifikationsniveau einhergeht, sondern dieser Zusammenhang durch die vorherrschende Geschlechterideologie hinsichtlich der Berufstätigkeit von Müttern moderiert wird. So bestehen in traditionellen Betriebskontexten trotz Frauenquote geschlechtsspezifische Einkommensungleichheiten, wohingegen in Betrieben mit egalitären Einstellungen keine Einkommensdisparitäten vorzufinden sind. Dagegen weisen die Ansichten bezüglich der gemeinsamen Erwirtschaftung des Haushaltseinkommens in der Belegschaft diesen Zusammenhang nicht auf; sowohl in traditionellen als auch in egalitären Betriebskontexten bestehen keine Einkommensungleichheiten zwischen Beschäftigten mit höherem Qualifikationsniveau. Ob die vorherrschenden Einstellungen in der Belegschaft die Assoziation der Frauenquote mit geschlechtsspezifischen Einkommensungleichheiten zwischen Beschäftigten mit höherem Qualifikationsniveau moderieren, ist folglich von der betrachteten Dimension der Geschlechterideologie abhängig. Demgegenüber weisen die vorherrschenden Geschlechterideologien in der Belegschaft keinen signifikanten Zusammenhang mit Gleichstellungsmaßnahmen und den Einkommensungleichheiten zwischen Beschäftigten mit geringerem Qualifikationsniveau auf. Zukünftige Forschung bedarf Längsschnittdaten, um zu untersuchen, ob Gleichstellungsmaß-

19 Weiterhin entfalten Mentoringprogramme ihre Wirksamkeit höchstwahrscheinlich erst einige Zeit nach der Implementierung. 
nahmen mit der Zeit ebenfalls in traditionellen Betriebskontexten ihre Wirkung entfalten.

Obwohl die LEEP-B3-Daten vielfältige Informationen sowohl auf der Beschäftigten- als auch auf der Betriebsebene enthalten, haben die diskutierten Ergebnisse Einschränkungen. Zum einen wurden nur Großbetriebe betrachtet. Wirkungsweisen von Mentoringprogrammen und Frauenquoten in kleinen und mittleren Betrieben könnten anders ausfallen. Zum anderen lassen Querschnittsdaten nur Aufschlüsse über statistische Korrelationen und keine kausalen Interpretationen zu. Aufgrund möglicher umgekehrter Kausalität kann nicht ausgeschlossen werden, dass Gleichstellungsmaßnahmen eher in Betrieben implementiert werden, die Frauen und Männer generell gleicher entlohnen. Die Berücksichtigung bestehender Geschlechterideologien legt jedoch nahe, dass dies nicht immer der Fall ist, da auch in Betrieben mit Gleichstellungsmaßnahmen traditionellere Geschlechterideologien und Einkommensungleichheiten vorzufinden sind. Zukünftige Längsschnittuntersuchungen, die Informationen über die Lohnlücken vor und nach der Implementierung beinhalten, sind notwendig, um die Effektivität von Gleichstellungsmaßnahmen im Zeitverlauf besser zu verstehen. Denn zum einen kann angenommen werden, dass direkt nach der Implementierung die stärksten Effekte vorzufinden sind, da ihnen viel Aufmerksamkeit und eine hohe Priorität beigemessen wird. Zum anderen kann vermutet werden, dass die Effektivität aufgrund der Erfahrungswerte und somit mit der Länge der Implementierung steigt.

Die Ergebnisse haben politische Implikationen. Sie legen nahe, dass die gesetzliche Frauenquote ein wichtiges Instrument zur Gleichstellung von Männern und Frauen am Arbeitsmarkt ist und auch auf Positionen unterhalb der obersten Ebene der Betriebshierarchie ausgeweitet werden sollte. Denn so können geschlechtsspezifische Einkommensungleichheiten innerhalb von Betrieben abgebaut werden. Weiterhin ist die Förderung der Gleichstellung von Frauen und Männern in der Familienpolitik, z. B. durch die Abschaffung des Ehegattensplittings und die Ausweitung der Paarmonate, wesentlich, um egalitäre Geschlechternormen in der Gesellschaft zu fördern. Diese egalitären Geschlechterideologien können sich wiederum positiv auf die Erfolgswahrscheinlichkeit von Gleichstellungsmaßnahmen, wie der Frauenquote, auswirken.

Förderung Die Forschung wurde gefördert durch die Deutsche Forschungsgemeinschaft als Teil des Projektes „Organisationale Ungleichheiten und Wechselwirkungen zwischen Verwirklichungschancen im Berufs- und Privatleben“, Fördernummer 373090005.

Funding Open Access funding provided by Projekt DEAL.

Open Access Dieser Artikel wird unter der Creative Commons Namensnennung 4.0 International Lizenz veröffentlicht, welche die Nutzung, Vervielfältigung, Bearbeitung, Verbreitung und Wiedergabe in jeglichem Medium und Format erlaubt, sofern Sie den/die ursprünglichen Autor(en) und die Quelle ordnungsgemäß nennen, einen Link zur Creative Commons Lizenz beifügen und angeben, ob Änderungen vorgenommen wurden.

Die in diesem Artikel enthaltenen Bilder und sonstiges Drittmaterial unterliegen ebenfalls der genannten Creative Commons Lizenz, sofern sich aus der Abbildungslegende nichts anderes ergibt. Sofern das betreffende Material nicht unter der genannten Creative Commons Lizenz steht und die betreffende Handlung nicht nach gesetzlichen Vorschriften erlaubt ist, ist für die oben aufgeführten Weiterverwendungen des Materials die Einwilligung des jeweiligen Rechteinhabers einzuholen. 
Weitere Details zur Lizenz entnehmen Sie bitte der Lizenzinformation auf http://creativecommons.org/ licenses/by/4.0/deed.de.

\section{Anhang}

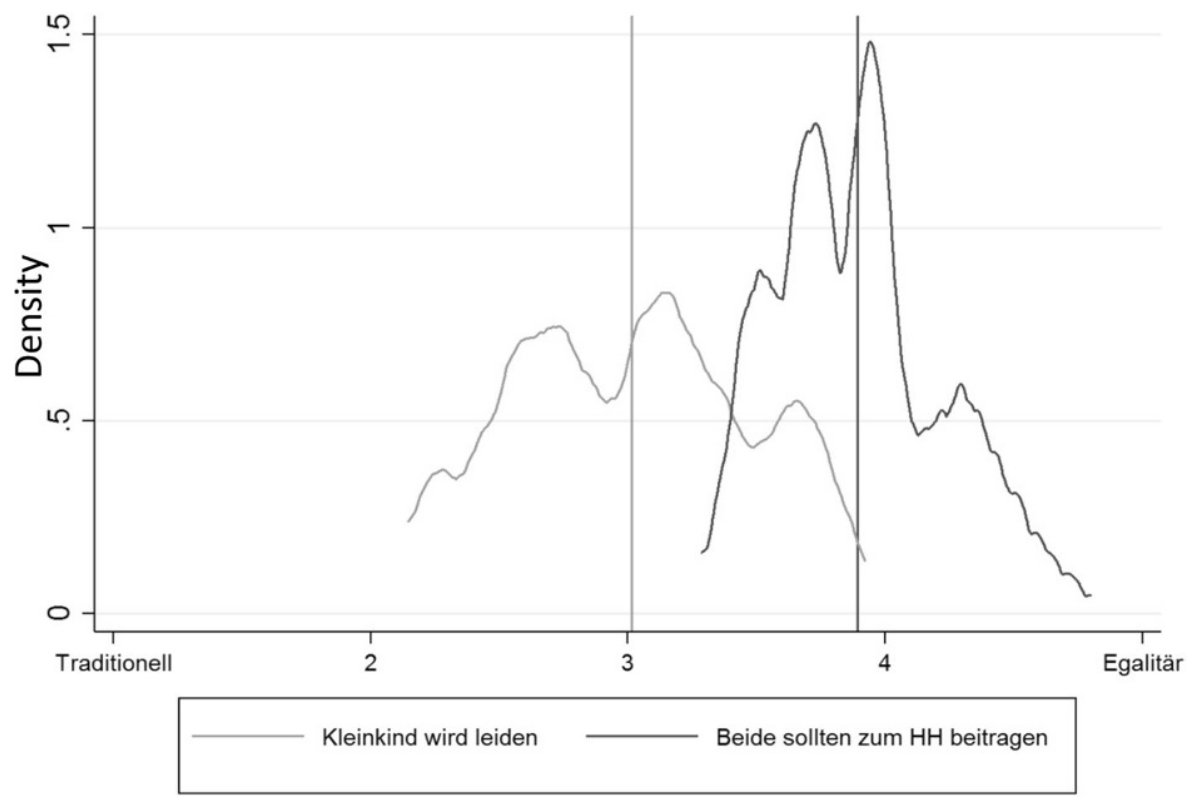

Abb. 4 Kernel-Density-Schätzer der durchschnittlichen Zustimmung zu Kleinkind wird unter berufstätiger Mutter leiden und Mann und Frau sollten zum Haushaltseinkommen beitragen in der Belegschaft (Die vertikalen Linien zeigen die durchschnittliche Zustimmung zu den jeweiligen Items in der Belegschaft über alle Betriebe) 


\section{Literatur}

Abadie, Alberto, Susan Athey, Guido W. Imbens und Jeffrey Wooldridge. 2017. When Should You Adjust Standard Errors for Clustering? NBER Working Paper 24003.

Abendroth, Anja, Silvia Melzer, Peter Jacobebbinghaus und Fabienne Schlechter. 2014. Methodological Report Employee and Partner Surveys of the Linked Employer-Employee Panel (LEEPB3) in Project B3: "Interactions Between Capabilities in Work and Private Life: A Study of Employees in Different Work Organizations.” SFB 882 Technical Report Series, No. 12.

Abendroth, Anja, Silvia Melzer, Alexandra Kalev und Donald Tomaskovic-Devey. 2017. Women's Access to Power and the Gender Earnings Gap. ILR Review 70(1):190-222.

Achatz, Juliane, Hermann Gartner und Timea Glück. 2005. Bonus oder Bias? Mechanismen geschlechtsspezifischer Entlohnung. Kölner Zeitschrift für Sozialpsychologie 57:466-493.

Acker, Joan. 1990. Hierarchies, jobs, bodies: A theory of gendered organizations. Gender \& Society 4(2):139-158.

Acker, Joan. 2006. Inequality Regimes: Gender, Class, and Race in Organizations. Gender \& Society 20(4):441-464.

Acock, Alan C. 2016. A Gentle Introduction to Stata. Stata. College Station, TX: Stata Press.

Aigner, Dennis J., und Glen G. Cain. 1977. Statistical Theories of Discrimination in Labor Markets. Industrial and Labor Relations Review 30(2):175-87.

Allport, Gordon W. 1954. The Nature of Prejudice. Boston: Addison-Wesley.

Aretz, Hans-Jürgen, und Katrin Hansen. 2003. Erfolgreiches Management von Diversity. Die multikulturelle Organisation als Strategie zur Verbesserung einer nachhaltigen Wettbewerbsfähigkeit. Zeitschrift für Personalforschung 17(1):9-36.

Arrow, Kenneth J. 1973. The Theory of Discrimination. In Discrimination in Labor Markets, Hrsg. Orley Ashenfelter und Albert Rees, 3-33. Princeton, NJ: Princeton University Press.

Arulampalam, Wiji, Alison L. Booth und Mark L. Bryan. 2007. Is There a Glass Ceiling over Europe? Exploring the Gender Pay Gap across the Wage Distribution. ILR Review 60(2):163-186.

Avent-Holt, Dustin, und Donald Tomaskovic-Devey. 2014. A Relational Theory of Earnings Inequality. American Behavioral Scientist 58(3):379-399.

Baron, James N., und William T. Bielby. 1980. Bringing the Firms Back in. American Sociological Review 45(5):737.

Becker, Gary Stanley. 1957. The economics of discrimination. Chicago: University of Chicago Press.

Berrey, Ellen. 2014. Breaking Glass Ceilings, Ignoring Dirty Floors: The Culture and Class Bias of Diversity Management. American Behavioral Scientist 58(2):347-370.

Bertrand, Marianne, Sandra E. Black, Sissel Jensen und Adriana Lleras-Muney. 2018. Breaking the Glass Ceiling? The Effect of Board Quotas on Female Labor Market Outcomes in Norway. The Review of Economic Studies 86(1):191-239.

Boll, Christina. 2010. Lohneinbußen von Frauen durch geburtsbedingte Erwerbsunterbrechungen. Wirtschaftsdienst 90(10):700-702.

Boll, Christina, Elisabeth Bublitz und Malte Hoffmann. 2015. Geschlechtsspezifische Berufswahl: Literatur- und Datenüberblick zu Einflussfaktoren, Anhaltspunkten struktureller Benachteiligung und Abbruchkosten. HWWI Policy Paper No. 90.

Brown, Deborah. 1986. The role of mentoring in the professional lives of university faculty women. Dissertation Abstracts International 47 (1):160A

Buche, Antje, Monika Jungbauer-Gans, Annekatrin Niebuhr und Cornelius Peters. 2013. Diversität und Erfolg von Organisationen. Zeitschrift für Soziologie 42(6):483-501.

Budig, Michelle J., und Paula England. 2001. The Wage Penalty for Motherhood. American Sociological Review 66(2):204-225.

Budig, Michelle J., Joya Misra und Irene Böckmann. 2012. The Motherhood Penalty in Cross-National Perspective: The Importance of Work-Family Policies and Cultural Attitudes. Social Politics 19(2):163-193.

Burke, Ronald J. 1984. Mentors in organizations. Group and Organization Studies 9(3):253-72.

Cardoso, Ana Rute, und Rudolf Winter-Ebmer. 2010. Female-led firms and gender wage policies. Industrial and Labor Realtions Review 64 (1):143-63.

Castilla, Emilio J. 2008. Gender, Race, and Meritocracy in Organizational Careers. American Journal of Sociology 113(6):1479-1526.

Cohen, Philip N., und Matt L. Huffman. 2007. Working for the woman? Female managers and the gender wage gap. American Sociological Review 72:681-704. 
Cohen, Lisa E., Joseph P. Broschak und Heather A. Haveman. 1998. And Then There Were More? The Effect of Organizational Sex Composition on the Hiring and Promoting of Managers. American Sociological Review 63:711-27.

Collins, Nancy W. 1983. Professional women and their mentors. Englewood Cliffs, New Jersey: Prentice Hall.

Cox, Taylor H. 1994. Cultural Diversity in Organizations: Theory, Research and Practice. San Francisco CA.: Berret-Koehler Publishers.

Cox, Taylor H., und Stacy Blake, 1991. Managing Cultural Diversity: Implications for Organizational Competitiveness. The Executive 5(3):45-56.

Davis, Shannon N., und Theodore N. Greenstein. 2009. Gender Ideology: Components, Predictors, and Consequences. Annual Review of Sociology 35:87-105.

Dechant, Anna, und Hans-Peter Blossfeld. 2015. Changes in the division of labor within highly educated German couples when the first child is born. Zeitschrift für Familienforschung 27(3):373-396.

Devine, Patricia G. 1989. Stereotypes and prejudice: Their automatic and controlled components. Journal of Personality and Social Psychology 56(1):5-18.

Diewald, Martin, Reinhard Schunck, Anja Abendroth, Silvia Melzer, Stephanie Pausch, Mareike Reimann, Björn Andernach und Peter Jacobebbinghaus. 2014. The SFB882-B3 Linked Employer-Employee Panel Survey (LEEP-B3). Schmollers Jahrbuch 134(3):379-89.

DiMaggio, Paul J., und Walter W. Powell. 1983. The Iron Cage Revisited: Institutional Isomorphism and Collective Rationality in Organizational Fields. American Sociological Review 48(2):147-160.

DiTomaso, Nancy. 2013. The American Non-Dilemma Racial Inequality Without Racism. New York: Russel Sage Foundation.

DiTomaso, Nancy, Corinne Post und Rochelle Parks-Yancy. 2007. Workforce Diversity and Inequality: Power, Status, and Numbers. Annual Review of Sociology 33:473-501.

Dobbin, Frank. 2009. Inventing Equal Opportunity. Princeton: Princeton University Press.

Dobbin, Frank, Daniel Schrage and Alexandra Kalev. 2015. Rage against the Iron Cage: The Varied Effects of Bureaucratic Personnel Reforms on Diversity. American Sociological Review 80 (5):1014-1044.

Dobbin, Frank., John R. Sutton, John W. Meyer und Richard Scott. 1993. Equal Opportunity Law and the Construction of Internal Labor Markets. American Journal of Sociology 99:396-427.

Dobbin, Frank, und Alexandra Kalev. 2016. Why diversity programs fail. Harvard Business Review.

Dreas, Susanne. 2019. Diversity Management in Organisationen der Sozialwirtschaft. Eine Einführung. Wiesbaden: VS Verlag für Sozialwissenschaften.

Ehrlich, Ulrike, Lara Minkus und Moritz Hess. 2019. Einkommensrisiko Pflege? Der Zusammenhang von familiärer Pflege und Lohn. Zeitschrift für Gerontologie und Geriatrie 52:1-7.

Ejrnœs, Mette, und Astrid Kunze. 2013. Work and Wage Dynamics around Childbirth. Scandinavian Journal of Economics 115:856-877.

Elliott, James R., und Ryan A. Smith. 2004. Race, Gender, and Workplace Power. American Sociological Review 69(3):365-386.

Ely, Robin J. 1995. The Power in Demography: Women's Social Constructions of Gender Identity at Work. The Academy of Management Journal 38(3):589-634.

Eurostat. 2019. Gender pay gap statistics. https://ec.europa.eu/eurostat/statistics-explained/index.php/ Gender_pay_gap_statistics (Zugegriffen: 16. Januar 2020).

Fiske, Susan T.1998. Stereotyping, prejudice, and discrimination. In The handbook of social psychology, Hrsg. Daniel T. Gilbert, Susan T Fiske und Gardner Lindzey, 357-411. Hoboken: Wiley.

Fitzenberger, Bernd, und Astrid Kunze. 2005. Vocational Training and Gender: Wages and Occupational Mobility Among Young Workers. ZEW Discussion Paper No. 05-66.

Fragenson, Ellen A. 1988. The power of a mentor: proteges' and non proteges' perceptions of their own power in organisations. Group \& Organisation studies 13 (2):182-192.

Frodermann, Corinna, Alexandra Schmucker und Dana Müller. 2018. Entgeltgleichheit zwischen Frauen und Männern in mittleren und großen Betrieben. IAB Forschungsbericht 3.

Fuchs, Michaela, Anja Rossen, Antje Weyh und Gabriele Wydra-Somaggio. 2019. Why do women earn more than men in some regions? Explaining regional differences in the gender pay gap in Germany. IAB Discussion Paper 11.

Gallego Granados, Patricia, und Katharina Wrohlich. 2018. Gender Pay Gap besonders groß bei niedrigen und hohen Löhnen. DIW-Wochenbericht 85(10):173-179.

Gangl, Markus. 2006. Scar Effects of Unemployment: An Assessment of Institutional Complementarities. American Sociological Review 71:986-1013. 
Gangl, Markus, und Andre Ziefle. 2009. Motherhood, Labor Force Behavior, and Women's Careers: An Empirical Assessment of the Wage Penalty for Motherhood in Britain, Germany, and the United States. Demography 46:341-369.

Ganzeboom, Harry. B. G., Paul M. De Graaf und Donal J. Treiman. 1992. A Standard International SocioEconomic Index of Occupational Status. Social Science Research 21(1):1-56.

Gartner, Herman, und Thomas Hinz. 2009. Geschlechtsspezifische Lohnungleichheit in Betrieben, Berufen und Jobzellen (1993-2006). Berliner Journal für Soziologie 19:557-575.

Giesecke, Johannes, und Roland Verwiebe. 2016. The Increase in Wage Inequality in Germany: New Findings for the Period Between 1998 and 2005. Zeitschrift für Soziologie 37(5):403-422.

Grunow. Daniela, Katia Begall und Sandra Buchler. 2018. Gender Ideologies in Europe: A Multidimensional Framework. Journal of Family and Marriage 80(1):42-60.

Heinze, Anja, und Elke Wolf. 2010. The intra-firm gender wage gap: a new view on wage differentials based on linked employer-employee data. Population Economics 23(3):851-879.

Helbig, Marcel. 2012. Die Umkehrung - Geschlechterungleichgheiten beim Erwerb des Abiturs im Wandel. In Soziologische Bildungsforschung, Hrsg. Rolf Becker und Heike Solga, 374-392. Wiesbaden: Springer VS.

Herring, Cedric. 2009. Does Diversity Pay? Race, Gender, and the Business Case for Diversity. American Sociological Review 74(2):208-224.

Hinz, Thomas. 2009. Mehrebenenanalyse. In Handbuch der Organisationsforschung, Hrsg. Stefan Kühl, Petra Strotdholz und Andreas Taffertshofer, 648-667. Wiesbaden: VS Verlag.

Hirsch, Boris. 2013. The impact of female managers on the gender pay gap: Evidence from linked employer-employee data for Germany. Economic letters 119(3): 348-350.

Holst, Elke, und Anita Wiemer. 2010. Frauen sind in Spitzengremien der Wirtschaft unterrepräsentiert. Eine Analyse der Ursachen und Handlungsansätze. Wirtschaftsdienst 90(10):692-699.

Holst, Elke, und Kathrin Wrohlich. 2019. Increasing Number of Women on Supervisory Boards of Major Companies in Germany: Executive Boards Still Dominated by Men. DIW Weekly Report 3:17-32.

Hoque, Kim, und Mike Noon. 2004. Equal Opportunities Policy and Practice in Britain. Work, Employment and Society 18(3):481-506.

Hox, Joop J. 2010. Multilevel Analysis: Techniques and Applications. New York, NY: Routledge.

Huffman, Matt. L., Joe King und Malte Reichelt. 2017. Equality for Whom? Organizational Policies and the Gender Gap across the German Earnings Distribution. ILR Review 70(1):16-41.

Hultin, Mia, und Ryszard Szulkin. 1999. Wages and unequal access to organizational power: An empirical test of gender discrimination. Administrative Science Quarterly 44 (3):453-72.

Ibarra, Herminia. 1992. Homophily and differential returns: Sex differences in network structure and access in an advertising firm. Administrative Science Quarterly 37(3): 422-447.

Ibarra, Herminia, Nancy M. Carter und Christine Silva, 2010. Why men still get more promotions than women. Harvard Business Review 88(9):80-85.

Iseke, Anja, und Kerstin Pull. 2017. Weibliche Nachwuchskräfte gewinnen? Mit Geschlechterstereotypen brechen! Personalquarterly 69:17-21.

Jüngling, Christina, und Daniela Rastetter. 2011. Die Implementierung von Gleichstellungsmaßnahmen. Optionen, Widerstände und Erfolgsstrategien. In Chancengleichheit durch Personalpolitik: Gleichstellung von Frauen und Männern in Unternehmen und Verwaltungen, Hrsg. Gertraude Krell, 127-140. Heidelberg: Springer Verlag.

Kaiser, Cheryl R., Brenda Major, Ines Jurcevic, Tessa L. Dover, Laura M. Brady und Jenessa R. Shapiro. 2013. Presumed fair: Ironic effects of organizational diversity structures. Journal of Personality and Social Psychology 104:504-519.

Kaiser-Belz, Manuela. 2008. Mentoring im Spannungsfeld von Personalentwicklung und Frauenförderung. Eine gleichstellungspolitische Maßnahme im Kontext beruflicher Felder. Wiesbaden: VS Verlag für Sozialwissenschaften.

Kalev, Alexandra. 2009. Cracking the Glass Cages? Restructuring and Ascriptive Inequality at Work. American Journal of Sociology 114(6):1591-1643.

Kalev, Alexandra, Frank Dobbin und Erin Kelly. 2006. Best Practices or Best Guesses? American Sociological Review 71(4):589-617.

Kandola, Rajvinder, und Johanna Fullerton. 1994. Managing the Mosaic: Diversity in Action. Institute of Personnel and Development.

Kanter, Rosabeth Moss. 1977. Men and Women of the Corporation. New York: Basic Books.

Kaplan, David. 2006. Can Diversity Training Discriminate? Backlash to Lesbian, Gay, and Bisexual Diversity Initiatives. Employee Responsibilities and Rights Journal 18(1):61-72. 
Kelly, Erin, und Frank Dobbin. 1999. Civil Rights Law at Work: Sex Discrimination and the Rise of Maternity Leave Policies. American Journal of Sociology 105(2):455-492.

Kidder, Deborah, Melenie Lankau, Donna Chrobat-Mason, Kelly A. Mollica und Ray Friedman. 2004. Backlash toward diversity initiatives: Examining the impact of diversity program justification, personal and group outcomes. The International Journal of conflict management 15 (1):77-102.

Kirton, Gill, und Anne-Marie Greene. 2005. The Dynamics of Managing Diversity: A critical Approach. Oxford: Elsevier Butterworth-Heinemann.

Knight, Carly R., und Mary C. Brinton. 2017. One Egalitarianism or Several? Two Decades of GenderRole Attitude Change in Europe. American Journal of Sociology 122(5):1485-1532.

Krell, Gertraude, und Barbara Sieben. 2011. Diversity Management: Chancengleichheit für alle und auch als Wettbewerbsvorteil. In Chancengleichheit durch Personalpolitik Gleichstellung von Frauen und Männern in Unternehmen und Verwaltungen, Hrsg. Gertraude Krell, Renate Ortlieb und Barbara Sieben, 155-174. Heidelberg: Springer Verlag.

Krishnan, Gopal V., und Linda M. Parsons. 2008. Getting to the Bottom Line: An Exploration of Gender and Earnings Quality. Journal of Business Ethics 78(1-2):65-76.

Kunze, Astrid. 2005. The evolution of the gender wage gap. Labour Economics 12:73-97.

Kurmeyer, Christine, und Dagmar Höppel. 2017. Mentoring in der Wissenschaft. In Praxishandbuch Mentoring in der Wissenschaft, Hrsg. Renate Petersen, Mechthild Budde, Pia Simone Brocke, Gitta Doebert und Helga Rudack, 17-26. Wiesbaden: Springer VS.

Kurtulus, Fidan Ana und Donald Tomaskovic-Devey. 2012. Do Female Top Managers Help Women to Advance? A Panel Study Using EEO-1 Records. The ANNALS of the American Academy of Political and Social Science 639(1):173-197.

Leuze, Kathrin, und Susanne Strauß. 2009. Lohnungleichheiten zwischen Akademikerinnen und Akademikern: Der Einfluss von fachlicher Spezialisierung, frauendominierten Fächern und beruflicher Segregation. Zeitschrift für Soziologie 38 (4):262-281.

Lockwood, Penelope. 2006. "Someone Like Me can be Successful": Do College Students Need SameGender Role Models? Psychology of Women Quarterly 30(1):36-46.

Ludsteck, Johannes. 2014. The Impact of Segregation and Sorting on the Gender Wage Gap: Evidence from German Linked Longitudinal Employer-Employee Data. ILR Review 67(2):362-394.

Mandel, Hadas, und Moshe Semyonov. 2006. A Welfare State Paradox: State Interventions and Women's Employment Opportunities in 22 Countries. American Journal of Sociology 111(6):1910-1949.

Maume, David J., Orlaith Heymann und Leah Ruppaner. 2019. National Board Quotas and the Gender Pay Gap among European Managers. Work, Employment and Society 33(6):1002-1019.

McPherson, Miller, Lynn Smith-Lovin und James M. Cook. 2001. Birds of a Feather: Homophily in Social Networks. Annual Review of Sociology 27:415-444.

Merklein, Alexander. 2017. Diversity Management in Deutschland. Empirische Untersuchung von Maßnahmen für Geschlecht und sexuelle Identität. Wiesbaden: Springer Gabler.

Meyer, John W., und Brian Rowan. 1977. Institutionalized Organizations: Formal Structure as Myth and Ceremony. American Journal of Sociology 83(2):340-363.

Mun, Eunmi, und Jiwook Jung. 2017. Change above the Glass Ceiling: Corporate Social Responsibility and Gender Diversity in Japanese Firms. Administrative Science Quarterly 63(2):409-440.

Naff, Katherine C., und J. Edward Kellough. 2003. Ensuring Employment Equity. International Journal of Public Administration 26(12):1307-1336.

Graf, Nele, und Frank Edelkraut. 2017. Mentoring. Das Praxisbuch für Personalverantwortliche und Unternehmer. Wiesbaden: Springer VS.

Nisic, Natascha, 2017. Smaller Differences in Bigger Cities? Assessing the Regional Dimension of the Gender Wage Gap. European Sociological Review 33(2):292-304.

Ohlendieck, Lutz. 2003. Die Anatomie des Glashauses. Ein Beitrag zum Verständnis des glass ceiling Phänomens. In Gender-from costs to benefits, Hrsg. Ursula Pasero, 183-193. Wiesbaden: Westdeutscher Verlag.

Pausch Stephanie, Julia Harand, Reinhard Schunck und Peter Jacobebbinghaus. 2014. Technical report employer survey Project B3: Interactions between capabilities in work and private life. SFB 882 Technical Report Series. Vol 4. Bielefeld: DFG Research Center (SFB) 882 From Heterogeneities to Inequalities.

Pettigrew, Thomas Fraser. 1998. Intergroup contact theory. Annual review of psychology 49:65-85.

Pettigrew, Thomas Fraser, und Linda R. Tropp. 2006. A meta-analytic test of intergroup contact theory. Journal of Personality and Social Psychology 90(5):751-783.

Phleps, Edmund. 1972. The Statistical Theory of Racism and Sexism. The American Economic Review 62(4):659-661. 
Rabe-Hesketh, Sophia, und Anders Skrondal. 2012. Multilevel and longitudinal modeling using stata (Third edition). College Station, Texas: StataCorp LP.

Ragins, Belle Rose. 1989. Barriers to mentoring: The female manager's dilemma. Human Relations 42(1):1-22.

Ragins, Belle Rose, und John L. Cotton. 1999. Mentor Functions and Outcomes: A Comparison of Men and Women in Formal and Informal Mentoring Relationships. Journal of Applied Psychology 84(4):529-50.

Redmond, Paul, und Seamus McGuinness., 2018. The Gender Wage Gap in Europe: Job Preferences, Gender Convergence and Distributional Effects. Oxford Bulletin of Economics and Statistics 81(3):564-587.

Reskin, Barbara F. 2000. Getting it Right: Sex and Race Inequality in Work Organizations. Annual Review of Sociology 26:707-709.

Ridgeway, Cecilia L. 2011. Framed by Gender. How Gender Inequality Persists in the Modern World. Oxford: Oxford University Press.

Ridgeway, Cecilia. L., und Shelley J. Correll. 2004. Unpacking the Gender System: A Theoretical Perspective on Gender Beliefs and Social Relations. Gender \& Society 18(4):510-531.

Ridgeway, Cecilia L., Elizabeth Heger Boyle, Kathy J. Kuipers, und Dawn T. Robinson. 1998. How Do Status Beliefs Develop? The Role of Resources and Interactional Experience. American Sociological Review 63:331-350.

Risman, Barbara J., und Georgiann Davis. 2013. From sex roles to gender structure. Current Sociology 61(5-6):733-755.

Rivera, Lauren. A. 2012. Hiring as Cultural Matching. American Sociological Review 77(6):999-1022.

Scandura, Terri A. 1992. Mentorship and career mobility: An empirical investigation. Journal of Organizational Behavior 13(2):169-174.

Scarborough, William J., Danny L. Lambouths und Allyson Holbrook. 2019. Support of workplace diversity policies: The role of race, gender, and beliefs about inequality. Social Science Research 79:194-210.

Singh, Val, Susan Vinnicombe und Kim Turnbull James. 2006. Constructing a professional identity: How young female managers use role models. Women in Management Review 21:67-81.

Stainback, Kevin., Sibyl Kleiner und Sheryl Skaggs. 2016. Women in Power: Undoing or Redoing the Gendered Organization? Gender \& Society 30(1):109-135.

Statistisches Bundesamt. 2018a. Erwerbstätigenquoten 1991 bis 2017. https://www.destatis.de/DE/ Themen/Arbeit/Arbeitsmarkt/Erwerbstaetigkeit/Tabellen/erwerbstaetigenquoten-gebietsstandgeschlecht-altergruppe-mikrozensus.html (Zugegriffen: 19. Jan. 2020).

Statistisches Bundesamt. 2018b. Frauenanteile nach akademischer Laufbahn. https://www.destatis.de/ DE/Themen/Gesellschaft-Umwelt/Bildung-Forschung-Kultur/Hochschulen/Tabellen/frauenanteileakademischelaufbahn.html (Zugegriffen: 19. Jan. 2020).

Statistisches Bundesamt. 2018c. Anteil der Akademikerinnen bei 30- bis 34-Jährigen doppelt so hoch wie vor einer Generation. https://www.destatis.de/DE/Presse/Pressemitteilungen/2018/09/PD18_332_ 217.html (Zugegriffen: 16. Januar 2020).

Steffes, Susanne, Annette Hillerich, Corinna Frodermann und Peter Krauss-Hoffman. 2017. Chancengleichheit von Frauen und Männern am Arbeitsplatz. Aktuelle Ergebnisse einer Betriebs- und Beschäftigtenbefragung. https://www.bmas.de/SharedDocs/Downloads/DE/PDF-Publikationen/a881monitor-chancengleichheit-von-frauen-und-maennern-am-arbeitsplatz.pdf?_blob=publicationFile $\& v=1$.pdf (Zugegriffen: 16. Januar 2020).

Stojmenovska, Dragana. 2019. Management gender composition and the gender pay gap: Evidence from British panel data. Gender, Work \& Organization 26(5):738-764.

Tajfel, Henri, und John Turner. 1979. An Integrative Theory of Intergroup Conflict. In Organizational Identity, Hrsg. Mary Jo Hatch und Majken Schultz, 56-66. Oxford: Oxford University Press.

Taylor, Shelley, Susan T. Fiske, Nany L. Etcoff und Audrey J. Ruderman. 1978. Categorical and Contextual Bases of Person Memory and Stereotyping. Journal of Personality and Social Psychology 36(7):778-793.

Tilly, Charles. 1998. Durable Inequality. Berkeley: University of California Press.

Tomaskovic-Devey, Donald, und Dustin Avent-Holt. 2019. Relational Inequalities. An Organizational Approach. Oxford: Oxford University Press.

Trappe, Heike, und Rachel Rosenfeld. 2004. Occupational Sex Segregation and Family Formation in the Former East and West Germany. Work and Occupations 31:155-192. 
Whitely, William, Thomas Dougherty und Georg F. Dreher. 1991. The relationship of mentoring and socioeconomic origin to managers' and professionals' early career progress. Academy of Management Journal 34(2):331-351.

Wrench, John. 2007. Diversity management and discrimination. Aldershot: Ashgate.

Yu, Wei-hsin, und Pei-lin Lee. 2013. Decomposing Gender Beliefs: Cross-National Differences in Attitudes Toward Maternal Employment and Gender Equality at Home. Sociological Inquiry 83(4):591-621.

Şherif, Muzafe. 1966. In common predicament. Social psychology of intergroup conflict and cooperation. Boston: Houghton Mifflin Company.

Eileen Peters 1991, Wissenschaftliche Mitarbeiterin an der Universität Bielefeld. Forschungsgebiete: Sozialstruktur- und Ungleichheitsforschung, Organisationale Ungleichheiten, Geschlechter- und Arbeitsmarktsoziologie.

Jil Drobe 1991, Workplace Development Managerin bei Heroal in Verl. Forschungsgebiete: Sozialstruktur- und Ungleichheitsforschung, Geschlechter- und Organisationssoziologie, digitalisierte Arbeitswelten.

Anja Abendroth Juniorprofessorin für technischen und sozialen Wandel an der Universität Bielefeld. Forschungsgebiete: Organisationale Ungleichheiten, Digitale Zukunft, Vereinbarkeit von Familie und Beruf, Geschlechterungleichheiten. Veröffentlichungen: Auswirkungen von Teleheimarbeit auf geschlechtsspezifische Einkommensungleichheiten in Arbeitsorganisationen. Die Bedeutung unterschiedlicher Umsetzungsformen. Kölner Zeitschrift für Soziologie und Sozialpsychologie 71, 2019 (mit M. Diewald); Women at Work: Women's Access to Power and the Gender Earning Gap. ILR Review 70, 2017 (mit S. Melzer, A. Kalev und D. Tomaskovic-Devey). 